\title{
On Degree Properties of Crossing-Critical Families of Graphs*
}

\author{
Drago Bokal ${ }^{\dagger} \quad$ Mojca Bračič \\ Faculty of Natural Sciences and Mathematics \\ University of Maribor \\ Maribor, Slovenia \\ drago.bokal@um.si mojca.bracic@student.um.si \\ Marek Derňár $r^{\ddagger} \quad$ Petr Hliněný \\ Faculty of Informatics \\ Masaryk University \\ Brno, Czech Republic \\ m.dernar@gmail.com hlineny@fi.muni.cz
}

Submitted: Mar 27, 2018; Accepted: Jan 28, 2019; Published: Mar 22, 2019

(C) The authors. Released under the CC BY-ND license (International 4.0).

\begin{abstract}
Answering an open question from 2007, we construct infinite $k$-crossing-critical families of graphs that contain vertices of any prescribed odd degree, for any sufficiently large $k$. To answer this question, we introduce several properties of infinite families of graphs and operations on the families allowing us to obtain new families preserving those properties. This conceptual setup allows us to answer general questions on behaviour of degrees in crossing-critical graphs: we show that, for any set of integers $D$ such that $\min (D) \geqslant 3$ and $3,4 \in D$, and for any sufficiently large $k$, there exists a $k$-crossing-critical family such that the numbers in $D$ are precisely the vertex degrees that occur arbitrarily often in (large enough) graphs of this family. Furthermore, even if both $D$ and some average degree in the interval $(3,6)$ are prescribed, $k$-crossing-critical families exist for any sufficiently large $k$.
\end{abstract}

Mathematics Subject Classifications: 05C10, 05C62

*A short preliminary version of this paper has appeared at Graph Drawing 2015.

${ }^{\dagger}$ This research was supported by the internationalisation of Slovene higher education within the framework of the Operational Programme for Human Resources Development 2007-2013 and by the Slovenian Research Agency Research projects J1-8130, N1-0057, and L7-5459, and by the research programme P1-0297.

†This research was supported by the Czech Science Foundation under the project 17-00837S. 


\section{Introduction}

Reducing the number of crossings in a drawing of a graph is considered one of the most important drawing aesthetics. Consequently, a great deal of research work has been invested into understanding what forces the number of edge crossings in a drawing of the graph to be large. There exist strong quantitative lower bounds, such as the famous Crossing Lemma [1, 14]. However, the quantitative bounds typically show their strength only in dense graphs, while in the area of graph drawing, we often deal with graphs having few edges.

The reasons for sparse graphs to have many crossings in any drawing are structural (there is a lot of "nonplanarity" in them). These reasons can be understood via so called $k$-crossing-critical graphs, which are the subgraph-minimal graphs that require at least $k$ edge crossings (the "minimal obstructions"). While there are only two 1-crossingcritical graphs, up to subdivisions - the Kuratowski graphs $K_{5}$ and $K_{3,3}$-it has been known already since Širán̆'s [19] and Kochol's [13] constructions that, for any $k \geqslant 2$, the structure of $k$-crossing-critical graphs is quite rich and non-trivial.

Although 2-crossing-critical graphs can be efficiently (although not easily) characterized [5], a full description for any $k \geqslant 3$ is clearly out of our current reach. Consequently, research has focused on interesting properties shared by all $k$-crossing-critical graphs (for certain $k$ ); successful attempts include, e.g., [7, 8, 10, 12, 17]. While we would like to establish as many specific properties of crossing-critical graphs as possible, the reality unfortunately seems to be against it. Many desired and conjectured properties of crossing-critical graphs have already been disproved by often complex and sophisticated constructions showing the odd behaviour of crossing-critical families, e.g. [6, 9, 11, 18].

We study properties of infinite families of $k$-crossing-critical graphs, for fixed values of $k$, since sporadic "small" examples of $k$-crossing-critical graphs tend to behave very wildly for every $k>1$. Among the most studied such properties are those related to vertex degrees in the critical families, see $[3,6,8,11,18]$. Often the research focused on the average degree a $k$-crossing-critical family may have - this rational number clearly falls into the interval $[3,6]$ if we forbid degree- 2 vertices. It is now known [8] that the true values fall into the open interval $(3,6)$, and all the rational values in this interval can be achieved [3]. However, for a fixed $k$, one cannot come arbitrarily close to 6 [8].

In connection with the proof of bounded pathwidth for $k$-crossing-critical families $[9,10]$, it turned out to be a fundamental question whether $k$-crossing-critical graphs have maximum degree bounded in $k$. The somehow unexpected negative answer was given by Dvořák and Mohar [6]. In 2007, Bokal noted that all the known (by that time) constructions of infinite $k$-crossing-critical families seem to use only vertices of degrees $3,4,6$, and he asked what other degrees can occur frequently (see the definition in Section 2) in $k$-crossing-critical families. Shortly after that Hliněný extended his previous construction [9] to include an arbitrary combination of any even degrees [11], for sufficiently large $k$. The characterization of 2-crossing-critical graphs [5] implied that also vertices of degree 5 occur arbitrarily often in 2-crossing-critical graphs.

Though, [11] answered only the easier half of Bokal's question, and it remained a wide 
open problem of whether there exist infinite $k$-crossing-critical families whose members contain many vertices of odd degrees greater than 5 . Our joint investigation has recently led to an ultimate positive answer.

The contribution and new results of our paper can be summarized as follows:

- In Section 2, we review the tools which are commonly used in constructions of crossing-critical families.

- Section 3 presents the key new contribution - a construction of crossing-critical graphs with repeated occurrence of any prescribed odd vertex degree (Proposition 3.1 and Theorem 3.2).

- In Section 4, we combine the new construction of Section 3 with previously known constructions to prove the following: for any set of integers $D$ such that $\min (D)=3$ and $3,4 \in D$, and for all sufficiently large $k$, there exists an infinite $k$-crossingcritical family such that the numbers in $D$ are precisely the vertex degrees which occur frequently in this family (Theorem 4.3).

- We extend the previous results in Section 5 to include an exhaustive discussion of possible average vertex degrees attained by our degree-restricted crossing-critical families (Theorem 5.1).

- Then, in Section 6, we pay special attention to infinite families of 2-crossing-critical graphs and summarize an exhaustive survey of their degree-related properties. The full detailed proofs for this section can be found in the arXiv version:1803.10509.

- Finally, in concluding Section 7, we list some remaining interesting open questions.

\section{Preliminaries}

We consider finite multigraphs without loops by default (i.e., we allow multiple edges unless we explicitly call a graph simple), and use the standard graph terminology otherwise. The degree of a vertex $v$ in a graph $G$ is the number of edges of $G$ incident to $v$ (cf. multigraphs), and the average degree of $G$ is the average of all the vertex degrees of $G$.

\subsection{Crossing number}

In a drawing of a graph $G$, the vertices of $G$ are points and the edges are simple curves joining their endvertices. It is required that no edge passes through a vertex, and no three edges cross in a common point. The crossing number $\operatorname{cr}(G)$ of a graph $G$ is the minimum number of crossing points of edges in a drawing of $G$ in the plane. For $k \in \mathbb{N}$, we say that a graph $G$ is $k$-crossing-critical, if $\operatorname{cr}(G) \geqslant k$ but, for every edge $e$ of $G, \operatorname{cr}(G-e)<k$.

Note that a vertex of degree 2 in $G$ is not relevant for a drawing of $G$ and for the crossing number, and we will often replace such vertices by edges between their two neighbours. Since also vertices of degree 1 are irrelevant for the crossing number, it is quite common to assume minimum degree 3. 


\subsection{Degree-universality}

The following terms formalize a vague notion that a certain vertex degree occurs frequently or arbitrarily often in an infinite family. For a finite set $D \subseteq \mathbb{N}$, we say that a family of graphs $\mathcal{F}$ is $D$-universal, if and only if, for every integer $m$, there exists a graph $G \in \mathcal{F}$ such that, for every $d \in D, G$ has at least $m$ vertices of degree $d$. It follows easily that $\mathcal{F}$ has infinitely many such graphs.

Clearly, if $\mathcal{F}$ is $D$ universal and $D^{\prime} \subseteq D$, then $\mathcal{F}$ is also $D^{\prime}$-universal. The family of all sets $D$, for which a given $\mathcal{F}$ is $D$-universal, therefore forms a poset under relation $\subseteq$. Maximal elements of this poset are of particular interest, and for "well-behaved" $\mathcal{F}$, these maximal elements are finite and unique. We distinguish this case with the following definition: $\mathcal{F}$ is $D$-max-universal, if it is $D$-universal, there are only finitely many degrees appearing in graphs of $\mathcal{F}$ that are not in $D$, and there exists an integer $M$, such that any degree not in $D$ appears at most $M$ times in any graph of $\mathcal{F}$.

Note that if $\mathcal{F}$ is both $D$-max-universal and $D^{\prime}$-max-universal, then $D=D^{\prime}$. It can also be easily seen that if $\mathcal{F}$ is $D$-max-universal, then there exists infinite $\mathcal{F}^{\prime} \subseteq \mathcal{F}$ such that, for any $m$, every sufficiently large member of $\mathcal{F}^{\prime}$ has, for each $d \in D$, at least $m$ vertices of degree $d$. Though, we do not specifically mention this property in the formal definition.

\subsection{Tools for constructing crossing-critical graphs}

A principal tool used in construction of crossing-critical graphs are tiles. They were used already in the early papers on infinite families of crossing-critical graphs by Kochol [13] and Richter and Thomassen [17], although they were formalized only in the work of Pinontoan and Richter [15, 16], answering Salazar's question [18] on average degrees in infinite families of $k$-crossing-critical graphs. Bokal built upon these results to fully settle Salazar's question when combining tiles with zip product [3]. Also a recent result that all large 2-crossing-critical graphs are composed of large multi-sets of specific 42 tiles [5] demonstrates that tiles are intimately related to crossing-critical graphs. In this section, we summarize the known results from [3, 5, 15], which we need for our constructions.

Tiles are essentially graphs equipped with two sequences of vertices that are identified among tiles or within a tile in order to, respectively, form new tiles or tiled graphs. The tiles can be drawn in the unit square respecting the order of these sequences of vertices, thus providing special, restricted drawings of tiles. Due to the restriction, the crossing number of these special drawings is an upper bound to the crossing number of either underlying graphs, or the graphs obtained by identifying these specific vertices. The formal concepts allowing these operations are summarized in the following definition and the lemma immediately after it:

Definition 2.1. Let $\lambda=\left(\lambda_{1}, \ldots, \lambda_{l}\right)$ and $\rho=\left(\rho_{1}, \ldots, \rho_{r}\right)$ be two sequences of distinct vertices of a graph $G$, where no vertex of $G$ appears in both $\lambda$ and $\rho$.

1. For any sequence $\lambda$, let $\bar{\lambda}$ denote its reversed sequence.

2. A tile is a triple $T=(G, \lambda, \rho)$. 
3. The sequence of vertices $\lambda$ is called the left wall and the sequence of vertices $\rho$ is called the right wall of $T$.

4. A tile drawing of a tile $T=(G, \lambda, \rho)$ is a drawing of $G$ in unit square $[0,1] \times[0,1]$ such that:

- all vertices of the left wall are drawn in $\{0\} \times[0,1]$ and all vertices of the right wall are drawn in $\{1\} \times[0,1]$;

- the left wall and the right wall have both decreasing $y$-coordinates.

5. The tile crossing number $\operatorname{cr}(T)$ of a tile $T$ is the smallest crossing number over all tile drawings of $T$.

6. A tile $T=(G, \lambda, \rho)$ is compatible with a tile $T^{\prime}=\left(G^{\prime}, \lambda^{\prime}, \rho^{\prime}\right)$ if $|\rho|=\left|\lambda^{\prime}\right|$ and cyclically-compatible if it is compatible with itself.

7. A sequence of tiles $\left(T_{0}, \ldots, T_{m}\right)$ is compatible, if, for $i=0, \ldots, m-1$, tiles $T_{i}$ and $T_{i+1}$ are compatible. It is cyclically-compatible if also $T_{m}$ is compatible with $T_{0}$.

8. The join of two compatible tiles $T=(G, \lambda, \rho)$ and $T^{\prime}=\left(G^{\prime}, \lambda^{\prime}, \rho^{\prime}\right)$ is defined as the tile $T \otimes T^{\prime}=\left(G \otimes G^{\prime}, \lambda, \rho^{\prime}\right)$, where $G \otimes G^{\prime}$ represents the graph obtained from the union of graphs $G$ and $G^{\prime}$, by identifying, for $i=1, \ldots,|\rho|, \rho_{i}$ with $\lambda_{i}^{\prime}$. If a vertex of degree 2 is introduced, then all maximal paths whose internal vertices are all of degree 2 are contracted to a single edge. Introduced double edges are retained.

9. Since the operator $\otimes$ is associative, the join $\otimes \mathcal{T}$ of a compatible sequence of tiles $\mathcal{T}=\left(T_{0}, \ldots, T_{m}\right)$ is defined as $\otimes \mathcal{T}=T_{0} \otimes \ldots \otimes T_{m}$.

10. Let $T=(G, \lambda, \rho)$ be a cyclically-compatible tile. The cyclization $\circ T$ of a tile $T$ is the graph $G$ obtained by identifying, for $i=1, \ldots,|\lambda|, \lambda_{i}$ with $\rho_{i}$.

11. Let $\mathcal{T}=\left(T_{0}, \ldots, T_{m}\right)$ be a cyclically-compatible sequence of tiles with $T_{0}=(G, \lambda, \rho)$, $T_{m}=\left(G^{\prime}, \lambda^{\prime}, \rho^{\prime}\right)$. The cyclization of $\mathcal{T}$ is defined as $\circ \mathcal{T}=\circ\left(T_{0} \otimes \ldots \otimes T_{m}\right)$.

12. Let $T=(G, \lambda, \rho)$ be a tile. The right-inverted tile $T^{\uparrow}$ is the tile $(G, \lambda, \bar{\rho})$ and the leftinverted tile ${ }^{\uparrow} T$ is the tile $(G, \bar{\lambda}, \rho)$. The inverted tile of $T$ is the tile ${ }^{\uparrow} T^{\uparrow}=(G, \bar{\lambda}, \bar{\rho})$ and the reversed tile of $T$ is the tile $T^{\leftrightarrow}=(G, \rho, \lambda)$.

13. For a compatible sequence of tiles $\mathcal{T}$, the twist is $\mathcal{T}^{\downarrow}=\left(T_{0}, \ldots, T_{m}^{\mathfrak{1}}\right)$, and the $i$-cut of $\mathcal{T}$ is $\mathcal{T} / i=\left(T_{i+1}, \ldots, T_{m}, T_{0}, \ldots, T_{i-1}\right)$.

Lemma 2.2 ([15]). Let $T$ be a cyclically-compatible tile. Then, $\operatorname{cr}(\circ T) \leqslant \operatorname{tcr}(T)$. Let $\mathcal{T}=\left(T_{0}, \ldots, T_{m}\right)$ be a compatible sequence of tiles. Then, $\operatorname{tcr}(\otimes \mathcal{T}) \leqslant \sum_{i=0}^{m} \operatorname{tcr}\left(T_{i}\right)$.

The above Lemma applies without any information on the internal structure of the tiles. However, by exploiting their internal structure (planarity and enough connectivity), we can also prove a lower bound on the tile crossing number, which can, with sufficiently many tiles, be exploited for the lower bound on the crossing number of the graph resulting from the tile. Prerequisites for these applications are summarized in the following definition and applied in the theorem that follows. 
Definition 2.3. Let $T=(G, \lambda, \rho)$ be a tile. Then:

1. $T$ is connected if $G$ is connected.

2. $T$ is planar if $\operatorname{tcr}(T)=0$.

3. $T$ is perfect if the following holds:

$-|\lambda|=|\rho| ;$

- $G-\lambda$ and $G-\rho$ are connected;

- for every $v \in \lambda$ there is a path to the right wall $\rho$ in $G$ internally disjoint from $\lambda$ and for every $u \in \rho$ there is a path to the left wall $\lambda$ in $G$ internally disjoint from $\rho$;

- for every $0 \leqslant i<j \leqslant|\lambda|$, there is a pair of disjoint paths, one joining $\lambda_{i}$ and $\rho_{i}$, and the other joining $\lambda_{j}$ and $\rho_{j}$.

Theorem $2.4([3])$. Let $\mathcal{T}=\left(T_{0}, \ldots, T_{\ell}, \ldots, T_{m}\right)$ be a cyclically-compatible sequence of tiles. Assume that, for some integer $k \geqslant 0$, the following hold: $m \geqslant 4 k-2$ and, for every $i \in\{0, \ldots, m\} \backslash\{\ell\}, \operatorname{tcr}(\otimes(\mathcal{T} / i)) \geqslant k$, and the tile $T_{i}$ is a perfect planar tile. Then, $\operatorname{cr}(\circ \mathcal{T}) \geqslant k$.

This theorem can yield exact crossing number under the assumptions of the next corollary.

Corollary $2.5([3])$. Let $\mathcal{T}=\left(T_{0}, \ldots, T_{\ell}, \ldots, T_{m}\right)$ be a cyclically-compatible sequence of tiles and let $k=\min _{i \in\{0, \ldots, m\} \backslash\{\ell\}} \operatorname{tcr}(\otimes(\mathcal{T} / i))$. If $m \geqslant 4 k-2$ and, for every $i \in$ $\{0, \ldots, m\} \backslash\{\ell\}$, the tile $T_{i}$ is a perfect planar tile, then $\operatorname{cr}(\circ \mathcal{T})=k$.

Exact lower bounds facilitate establishing criticality of the tiles and graphs, as the smallest drop in crossing number suffices for criticality of an edge. For combinatorially handling the criticality of the constructed graph on the basis of the properties of tiles, we introduce degeneracy of tiles and criticality of sequences of tiles as follows:

\section{Definition 2.6.}

1. A tile $T$ is $k$-degenerate if it is perfect, planar and, for any $e \in E(T), \operatorname{tcr}\left(T^{\uparrow}-e\right)<k$.

2. A sequence $\mathcal{T}=\left(T_{0}, \ldots, T_{m}\right)$ is $k$-critical if, for every $i=0, \ldots, m$, the tile $T_{i}$ is $k$-degenerate and $\min _{i \in\{0, \ldots, m-1\}} \operatorname{tcr}\left(\otimes\left((\mathcal{T} / i)^{\uparrow}\right)\right) \geqslant k$.

Using these concepts, Corollary 2.5 can be applied to establish criticality of graphs resulting from crossing critical sequences of tiles or from degenerate tiles.

Corollary $2.7([3])$. Let $\mathcal{T}=\left(T_{0}, \ldots, T_{m}\right)$ be a $k$-critical sequence of tiles. Then, $T=\otimes \mathcal{T}$ is a $k$-degenerate tile. If $m \geqslant 4 k-2$ and $\mathcal{T}$ is cyclically-compatible, then $\circ\left(T^{\uparrow}\right)$ is a $k$ crossing-critical graph. 
To estimate the tile crossing number, we use an informal tool called gadget. This can be any structure inside of a tile $T$, which guarantees a certain number of crossings in every tile drawing of $T$. The gadgets we use are twisted pairs of paths, guaranteeing one crossing each, and staircase strips of width $n$, guaranteeing $\left(\begin{array}{l}n \\ 2\end{array}\right)-1$ crossings.

Definition 2.8. A traversing path in a tile $T=(G, \lambda, \rho)$ is a path $P$ in the graph $G$, for which there exist indices $i(P) \in\{1, \ldots,|\lambda|\}$, and $j(P) \in\{1, \ldots,|\rho|\}$, so that $P$ is a path from $\lambda(P)=\lambda_{i(P)}$ to $\rho(P)=\rho_{j(P)}$ and $\lambda(P)$ and $\rho(P)$ are the only wall vertices of $P$.

A pair of disjoint traversing paths $\{P, Q\}$ is twisted if $i(P)<i(Q)$ and $j(P)>j(Q)$, and aligned otherwise. A family $\mathcal{W}$ of pairs of disjoint traversing paths is aligned, if all the pairs in $\mathcal{W}$ are aligned. The family is twisted, if all the pairs are twisted.

The disjointness of a twisted pair $\{P, Q\}$ implies one crossing in any tile drawing of $T$. This is generalized to twisted families in the following lemma:

Lemma 2.9 ([3]). Let $\mathcal{W}$ be a twisted family in a tile $T$, such that no edge occurs in two distinct paths of $\cup \mathcal{W}$. Then, $\operatorname{tcr}(T) \geqslant|\mathcal{W}|$.

The following definition presents a staircase tile, adapted from [3]. Such detailed definition is needed as this tile is later used as a part of our new constructed tile defined in Section 3. A reader should understand it quickly when referring to Figure 1.

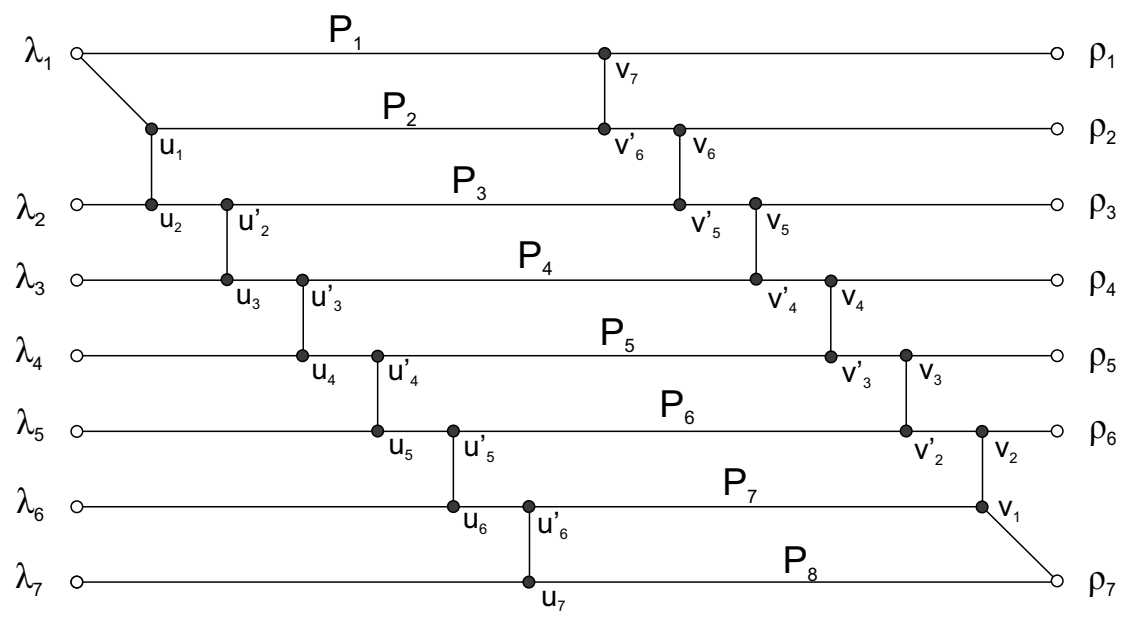

Figure 1: A staircase tile $S_{8}$. The wall vertices are drawn in white and internal vertices in black.

Definition 2.10 ([3]). Let $n \in \mathbb{N}, n \geqslant 3$. The staircase tile of width $n$ is a tile $S_{n}=$ $(G, \lambda, \rho)$ with $\lambda=\left(\lambda_{1}, \lambda_{2}, \ldots, \lambda_{n-1}\right), \rho=\left(\rho_{1}, \rho_{2}, \ldots, \rho_{n-1}\right)$ for which the following holds:

- $S_{n}$ consists of a sequence of traversing paths $\mathcal{P}=\left\{P_{1}, P_{2}, \ldots, P_{n}\right\}$ with the property:

$$
-\lambda\left(P_{1}\right)=\lambda_{1}, \rho\left(P_{1}\right)=\rho_{1} \text { and } \lambda\left(P_{n}\right)=\lambda_{n-1}, \rho\left(P_{n}\right)=\rho_{n-1},
$$


- for $i=2, \ldots, n-1, \lambda\left(P_{i}\right)=\lambda_{i-1}$ and $\rho\left(P_{i}\right)=\rho_{i}$.

- The only non-wall vertices of $S_{n}$ are, for $i=1, n-1, u_{i} \in P_{i+1}, v_{i} \in P_{n-i}$, and, for $i=2, \ldots, n-2, u_{i}, u_{i}^{\prime} \in P_{i+1}$ and $v_{i}, v_{i}^{\prime} \in P_{n-i}$.

- For $i=2,3, \ldots, n-2$, the position of a non-wall vertices of $S_{n}$ is such that $\left|e\left(u_{i}\right) \cap e\left(\lambda_{i}\right)\right|=1,\left|e\left(u_{i}\right) \cap e\left(u_{i}^{\prime}\right)\right|=1,\left|e\left(v_{i}\right) \cap e\left(\rho_{n-i}\right)\right|=1$ and $\left|e\left(v_{i}\right) \cap e\left(v_{i}^{\prime}\right)\right|=1$.

- The additional edges are $u_{1} u_{2}, v_{1} v_{2}$ and, for $i=2, \ldots, n-2, u_{i}^{\prime} u_{i+1}$ and $v_{i} v_{i+1}^{\prime}$.

For $n \geqslant 3$, a staircase tile $S_{n}$ is a perfect planar tile.

Definition 2.11 ([3]). Let $n \geqslant 3$ be integer and let $m \geqslant 3$ be an odd integer. The staircase sequence of length $m$ is defined as $\mathcal{S}_{n, m}=\left(S_{n},{ }^{\uparrow} S_{n}{ }^{\downarrow}, S_{n},{ }^{\uparrow} S_{n} \uparrow, \ldots,{ }^{\uparrow} S_{n}{ }^{\uparrow}, S_{n}\right)$ and the staircase strip graph as $S(n, m)=\circ\left(\mathcal{S}_{n, m}{ }^{\uparrow}\right)$.

Proposition 2.12 ([3]). The staircase strip graph, $S(n, m)$, of width $n$ and odd length $m \geqslant 4\left(\begin{array}{l}n \\ 2\end{array}\right)-5$ is a crossing-critical graph with $\operatorname{cr}(S(n, m))=\left(\begin{array}{l}n \\ 2\end{array}\right)-1$.

This concludes our discussion of known results on tiles in graphs. Tiled graphs are joined together using zip product construction $[2,3]$. We use the version restricted to vertices of degree three, as introduced in [11].

Definition 2.13. For $i \in\{1,2\}$, let $G_{i}$ be a graph and let $v_{i} \in V\left(G_{i}\right)$ be its vertex of degree 3 , such that $G_{i}-v_{i}$ is connected and $v_{i}$ is incident only to simple edges. We denote the neighbours of $v_{i}$ by $u_{j}^{i}$ for $j \in\{1,2,3\}$. The zip product of $G_{1}$ and $G_{2}$ according to vertices $v_{1}, v_{2}$ and their neighbours, is obtained from the disjoint union of $G_{1}-v_{1}$ and $G_{2}-v_{2}$ by adding three edges $u_{1}^{1} u_{1}^{2}, u_{2}^{1} u_{2}^{2}, u_{3}^{1} u_{3}^{2}$.

While crossing number is super-additive over general zip products only under a technical connectivity condition, the following theorem holds for zip products of degree (at most) three:

Theorem 2.14 ([4]). Let $G$ be a zip product of $G_{1}$ and $G_{2}$ as in Definition 2.13. Then, $\operatorname{cr}(G)=\operatorname{cr}\left(G_{1}\right)+\operatorname{cr}\left(G_{2}\right)$. Consequently, if, for $i=1,2, G_{i}$ is $k_{i}$-crossing-critical, then $G$ is $\left(k_{1}+k_{2}\right)$-crossing-critical.

\section{Crossing-Critical Families with High Odd Degrees}

We first present a new construction of a crossing-critical family containing many vertices of an arbitrarily prescribed odd degree (recall that the question of an existence of such families has been the main motivation for this research).

The construction defines a graph $G(\ell, n, m)$ with three integer parameters $\ell \geqslant 1, n \geqslant 3$ and odd $m \geqslant 3$, as follows. There is a tile $H_{\ell, n}$, with the walls of size $2 \ell+n-1$, which is illustrated in Figure 2. Formally, $H_{\ell, n}$ consists of $2 \ell+n$ pairwise edge disjoint paths, grouped into three families $P_{1}^{\prime}, \ldots, P_{\ell}^{\prime}, Q_{1}^{\prime}, \ldots, Q_{\ell}^{\prime}$, and $S_{1}^{\prime}, \ldots, S_{n}^{\prime}$, and an additional set $F^{\prime}$ of $2(n-2)$ edges not on these paths, see Figure 2 . We continue with more detailed definition: 


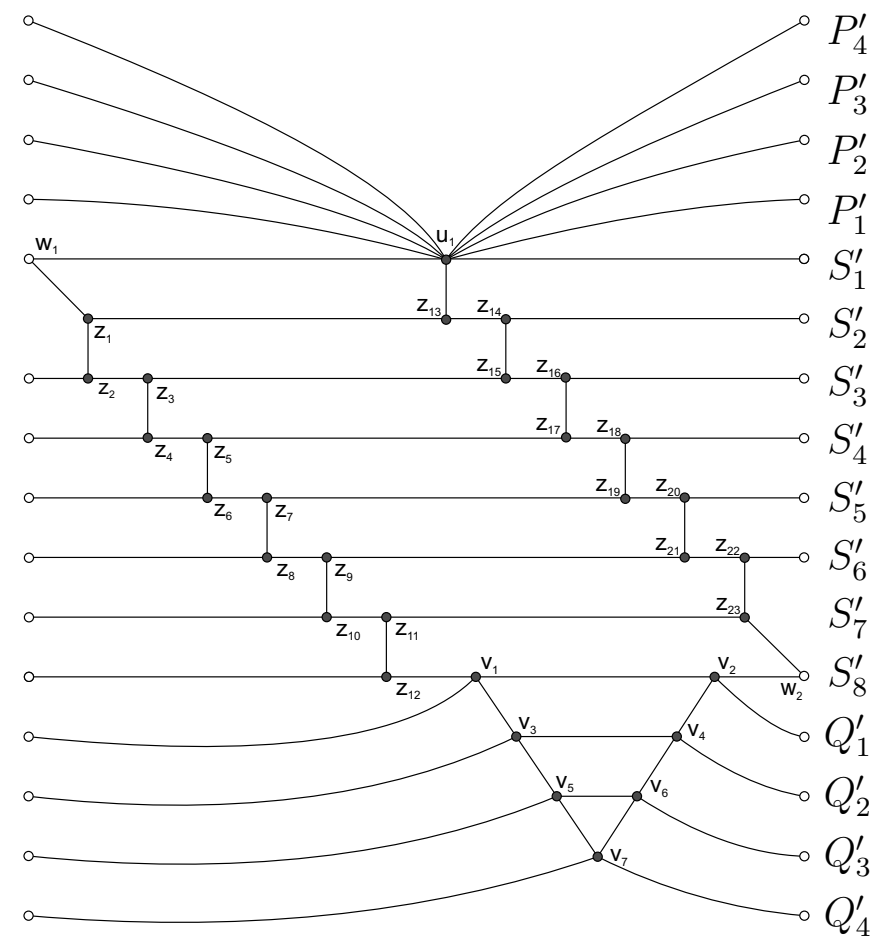

Figure 2: A tile drawing of $H_{4,8}$. The wall vertices are drawn in white and internal vertices in black.

- The paths $S_{1}^{\prime}, \ldots, S_{n}^{\prime}$ are pairwise vertex-disjoint except that $S_{1}^{\prime}$ shares one vertex with $S_{2}^{\prime}\left(w_{1}\right.$ in Figure 2) and $S_{n-1}^{\prime}$ shares one vertex with $S_{n}^{\prime}\left(w_{2}\right.$ in Figure 2). The additional 2 edges of $F^{\prime}$ appear between vertices of paths: $S_{2}^{\prime}$ and $S_{3}^{\prime}$ (edge $z_{1} z_{2}$ in Figure 2), $S_{1}^{\prime}$ and $S_{2}^{\prime}$ (edge $u_{1} z_{2(n-1)-1}$ in Figure 2). If $n>3$, then, for $i=2, \ldots, n-2$, additional $n-3$ edges of $F^{\prime}$ appear between vertices of paths $S_{i+1}^{\prime}$ and $S_{i+2}^{\prime}$ (edges $z_{2 i-1} z_{2 i}$ in Figure 2) and additional $n-3$ edges of $F^{\prime}$ appear between vertices of paths $S_{i}^{\prime}$ and $S_{i+1}^{\prime}$ (edges $z_{2(n-3)+2 i} z_{2(n-3)+2 i+1}$ in Figure 2).

- The union $S_{1}^{\prime} \cup \ldots \cup S_{n}^{\prime} \cup F^{\prime}$ is (consequently) a subdivision of the aforementioned staircase tile from Definition 2.10.

- Let $\lambda=\left(\lambda_{1}, \ldots, \lambda_{2 \ell+n-1}\right)$ be the left wall and $\rho=\left(\rho_{1}, \ldots, \rho_{2 \ell+n-1}\right)$ the right wall of $H_{\ell, n}$. The paths $P_{1}^{\prime}, \ldots, P_{\ell}^{\prime}$ are ordered such that, for $i=1, \ldots, n, \lambda\left(P_{i}\right)=\lambda_{\ell+1-i}$ and $\rho\left(P_{i}\right)=\rho_{\ell+1-i}$. The paths $Q_{1}^{\prime}, \ldots, Q_{\ell}^{\prime}$ are ordered such that, for $i=1, \ldots, n$, $\lambda\left(Q_{i}\right)=\lambda_{\ell+n-1+i}$ and $\rho\left(P_{i}\right)=\rho_{\ell+n-1+i}$.

- The paths $P_{1}^{\prime}, \ldots, P_{\ell}^{\prime}$ all share the top-most vertex $u_{1}$ of $S_{1}^{\prime}$.

- The paths $Q_{1}^{\prime}$ and $S_{n}^{\prime}$ shares exactly two vertices of degree $4\left(v_{1}\right.$ and $v_{2}$ in Figure 2$)$. For $i=1, \ldots, \ell-2, Q_{i}^{\prime}$ shares exactly two vertices with $Q_{i+1}^{\prime}$ and these shared vertices are of degree 4 , as depicted in Figure 2 (vertices $v_{i+2}, \ldots, v_{2 \ell-2}$ ). The paths $Q_{\ell-1}^{\prime}$ and $Q_{\ell}^{\prime}$ shares exactly one vertex of degree $4\left(v_{2 \ell-1}\right.$ in Figure 2$)$. 


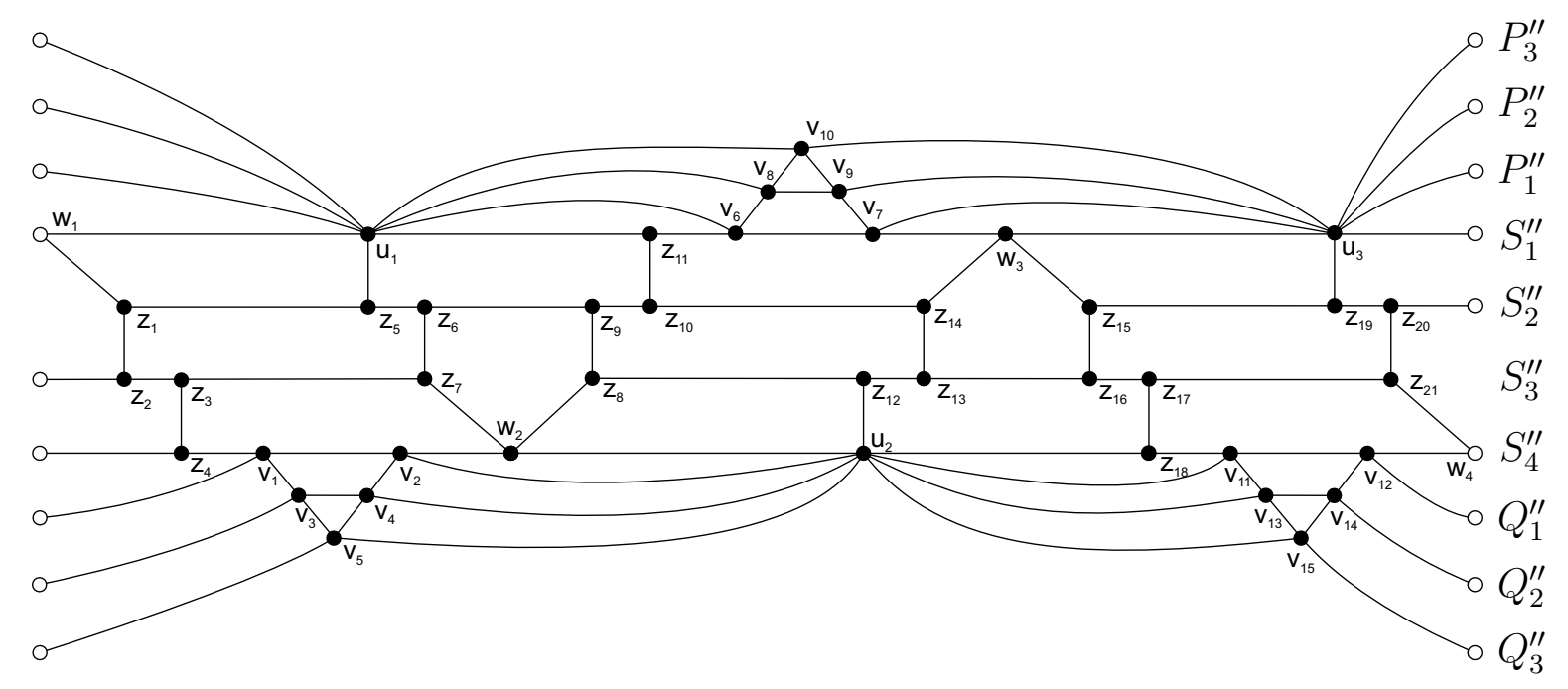

Figure 3: A tile drawing of $G_{3,4}$. The wall vertices are drawn in white and internal vertices in black.

Let $G_{\ell, n}$ be a tile composed of three copies of $H_{\ell, n}$ such that $G_{\ell, n}=H_{\ell, n} \otimes \mathfrak{} H_{\ell, n}{ }^{\uparrow} \otimes H_{\ell, n}$, which is illustrated in Figure 3. Let $\mathcal{G}(\ell, n, m)=\left(G_{\ell, n},{ }^{\uparrow} G_{\ell, n}{ }^{\uparrow}, G_{\ell, n} \ldots,{ }^{\uparrow} G_{\ell, n}{ }^{\uparrow}, G_{\ell, n}\right)$ be a sequence of such tiles of length $m$, and let $G(\ell, n, m)$ be constructed as the cyclization $\circ\left(\mathcal{G}(\ell, n, m)^{\uparrow}\right)$.

In the degenerate case of $\ell=0$, the graph $G(0, n, m)$ is a staircase strip graph (see Definition 2.11), and $G(0, n, m)$ will be contained in $G(\ell, n, m)$ as a subdivision for every $\ell$.

For $i=1, \ldots, \ell$ and $j=1, \ldots, n$, let $P_{i}^{\prime \prime}, Q_{i}^{\prime \prime}, S_{j}^{\prime \prime}$ denote the paths obtained by three copies of each of $P_{i}^{\prime}, Q_{i}^{\prime}, S_{i}^{\prime}$ from $H_{\ell, n}$ as $P_{i}^{\prime \prime}=\otimes\left(P_{i}^{\prime}, Q_{i}^{\prime}, P_{i}^{\prime}\right), Q_{i}^{\prime \prime}=\otimes\left(Q_{i}^{\prime}, P_{i}^{\prime}, Q_{i}^{\prime}\right)$ and $S_{j}^{\prime \prime}=\otimes\left(S_{j}^{\prime}, S_{n+1-j}^{\prime}, S_{j}^{\prime}\right)$. Then $P_{1}^{\prime \prime}, \ldots, P_{\ell}^{\prime \prime}, Q_{1}^{\prime \prime}, \ldots, Q_{\ell}^{\prime \prime}$, and $S_{1}^{\prime \prime}, \ldots, S_{n}^{\prime \prime}$ are all traversing paths of $G_{\ell, n}$.

For $i=1, \ldots, \ell$ and $j=1, \ldots, n$, let $\bar{P}_{i}, \bar{Q}_{i}, \bar{S}_{i}$ denote the paths which are obtained by $m$ copies of each of $P_{i}^{\prime \prime}, Q_{i}^{\prime \prime}, S_{i}^{\prime \prime}$ from $G_{\ell, n}$ as $\bar{P}_{i}=\otimes\left(P_{i}^{\prime \prime}, Q_{i}^{\prime \prime}, P_{i}^{\prime \prime}, \ldots, Q_{i}^{\prime \prime}, P_{i}^{\prime \prime}\right), \bar{Q}_{i}=$ $\otimes\left(Q_{i}^{\prime \prime}, P_{i}^{\prime \prime}, Q_{i}^{\prime \prime}, \ldots, P_{i}^{\prime \prime}, Q_{i}^{\prime \prime}\right)$ and $\bar{S}_{j}=\otimes\left(S_{j}^{\prime \prime}, S_{n+1-j}^{\prime \prime}, S_{j}^{\prime \prime}, \ldots, S_{n+1-j}^{\prime \prime}, S_{j}^{\prime \prime}\right)$. Then $\bar{P}_{i}, \bar{Q}_{i}, \bar{S}_{i}$ are all traversing paths of $\otimes \mathcal{G}(\ell, n, m)$.

The proof of the following basic properties is straightforward, as attentive reader could easily verify from the illustrating pictures of $H_{\ell, n}$ (recall that degree- 2 vertices are contracted in a tile join).

Proposition 3.1. For every $\ell \geqslant 1$ and $n \geqslant 3$, the tiles $H_{\ell, n}$, and hence also $G_{\ell, n}$, are perfect planar tiles. The graph $G(\ell, n, m)$ has $3 m(2 \ell+4 n-8)$ vertices, out of which $3 m \cdot 2 \ell$ have degree $4,3 m(4 n-9)$ have degree 3 , and remaining $3 m$ vertices have degree $2 \ell+3$. The average degree of $G(\ell, n, m)$ is

$$
\frac{5 l+6 n-12}{l+2 n-4} .
$$

We conclude with the main desired property of the graph $G(\ell, n, m)$. 
Theorem 3.2. Let $\ell \geqslant 1, n \geqslant 3$ be integers. Let $k=\left(\ell^{2}+\left(\begin{array}{l}n \\ 2\end{array}\right)-1+2 \ell(n-1)\right)$ and let $m \geqslant 4 k-1$ be odd. Then the graph $G(\ell, n, m)$ is $k$-crossing-critical.

Proof. By using Theorem 2.4 and symmetry, it suffices to prove the following:

I) $\operatorname{tcr}\left(\otimes \mathcal{G}(\ell, n, m)^{\uparrow}\right) \geqslant k$, and

II) every edge of $G_{\ell, n}$ corresponding to one copy of $H_{\ell, n}$ in it is critical, meaning that, for every edge $e \in E\left(H_{\ell, n}\right) \subseteq E\left(G_{\ell, n}\right), \operatorname{tcr}\left(G_{\ell, n} \uparrow-e\right)<k$.

Recall the pairwise edge-disjoint traversing paths $\bar{P}_{1}, \ldots, \bar{P}_{\ell}, \bar{Q}_{1}, \ldots, \bar{Q}_{\ell}$, and $\bar{S}_{1}, \ldots, \overline{S_{n}}$ of the join $\otimes \mathcal{G}(\ell, n, m)$. We define the following disjoint sets of pairs of these paths, such that each pair is formed by vertex-disjoint paths:

- $\mathcal{A}=\left\{\left\{\bar{P}_{i}, \bar{Q}_{j}\right\}: 1 \leqslant i, j \leqslant \ell\right\}$ where $|\mathcal{A}|=\ell^{2}$,

- $\mathcal{B}=\left\{\left\{\bar{P}_{i}, \bar{S}_{j}\right\}: 1 \leqslant i \leqslant \ell, 1<j \leqslant n\right\}$ where $|\mathcal{B}|=\ell(n-1)$,

- $\mathcal{C}=\left\{\left\{\bar{Q}_{i}, \bar{S}_{j}\right\}: 1 \leqslant i \leqslant \ell, 1 \leqslant j<n\right\}$ where $|\mathcal{C}|=\ell(n-1)$.

Each pair in $\mathcal{A} \cup \mathcal{B} \cup \mathcal{C}$ is twisted in $\otimes \mathcal{G}(\ell, n, m)^{\uparrow}$, and so these pairs account for at least $|\mathcal{A}|+|\mathcal{B}|+|\mathcal{C}|=2 \ell(n-1)+\ell^{2}$ crossings in a tile drawing of $\otimes \mathcal{G}(\ell, n, m)^{\uparrow}$, by Lemma 2.9. Importantly, each of these crossings involves at least one edge of $R=$ $\bar{P}_{1} \cup \ldots \cup \bar{P}_{\ell} \cup \bar{Q}_{1} \cup \ldots \cup \bar{Q}_{\ell}$. The subgraph $\otimes \mathcal{G}(\ell, n, m)-E(R)$ contains a subdivision of the staircase strip $\otimes \mathcal{G}(0, n, m)$.

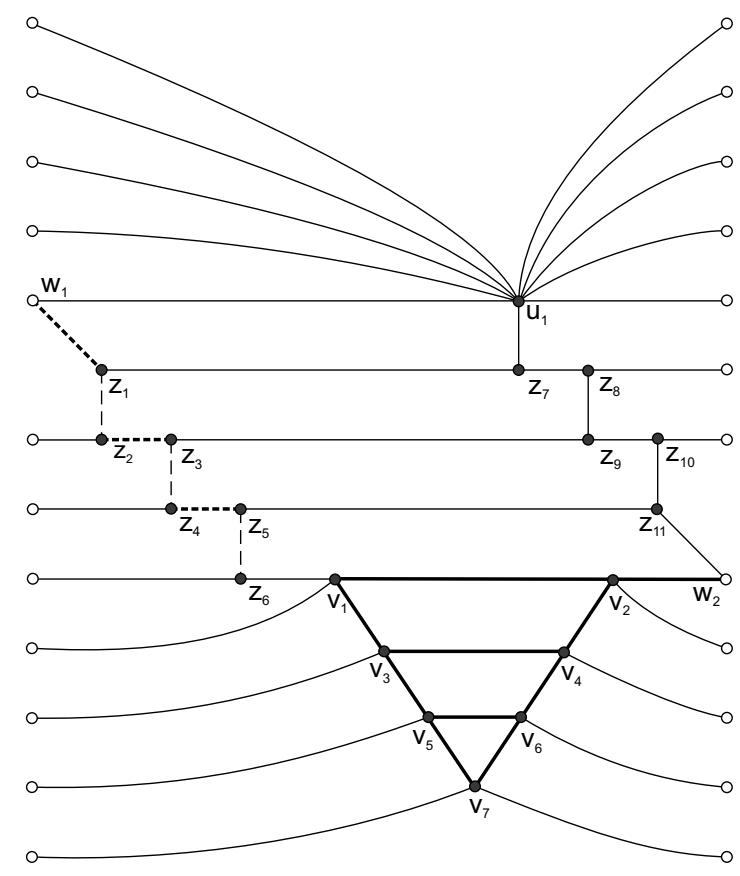

Figure 4: A tile drawing of $H_{4,5}$ depicted with 4 types of edges. 
Hence any tile drawing of $\otimes \mathcal{G}(\ell, n, m)^{\ddagger}$ contains at least another $\operatorname{tcr}\left(\otimes \mathcal{G}(0, n, m)^{\ddagger}\right)$ crossings not involving any edges of $R$. Since $\operatorname{tcr}\left(\otimes \mathcal{G}(0, n, m)^{\uparrow}\right)=\left(\begin{array}{l}n \\ 2\end{array}\right)-1$ by Proposition 2.12 , we get $\operatorname{tcr}\left(\otimes \mathcal{G}(\ell, n, m)^{\downarrow}\right) \geqslant\left(\begin{array}{l}n \\ 2\end{array}\right)-1+2 \ell(n-1)+\ell^{2}=k$, thus proving (I).

To prove (II), we investigate the tile drawing in Figure 5. It is routine to count that a natural generalization of this drawing has precisely $\left(\begin{array}{c}n-2 \\ 2\end{array}\right)+2(n-2)+2 \ell(n-1)+\ell^{2}=k$ crossings, and so it is optimal. Three types of crossings are presented in Figure 5, Figure 6, Figure 7 and Figure 8:

- grey triangles are the $\ell^{2}$ crossings of each pair in $\mathcal{A}$;

- grey 4-stars are the $2 \ell(n-1)$ crossings of each pair in $\mathcal{B} \cup \mathcal{C}$;

- grey squares are the $\left(\begin{array}{c}n-2 \\ 2\end{array}\right)+2(n-2)=\left(\begin{array}{c}n \\ 2\end{array}\right)-1$ crossings of edges in a staircase part, $G(0, n, m)$, of a graph $G(\ell, n, m)$. The $\left(\begin{array}{c}n-2 \\ 2\end{array}\right)$ crossings appear in the middle of Figure 5 caused by edges, for $i=1, \ldots, n-2, z_{2 i-1} z_{2 n+2 i-5}$. The $n-2$ crossings are caused by edge $w_{1} u_{1}$ and $n-2$ crossings by edge $z_{2 n-4} w_{2}$.

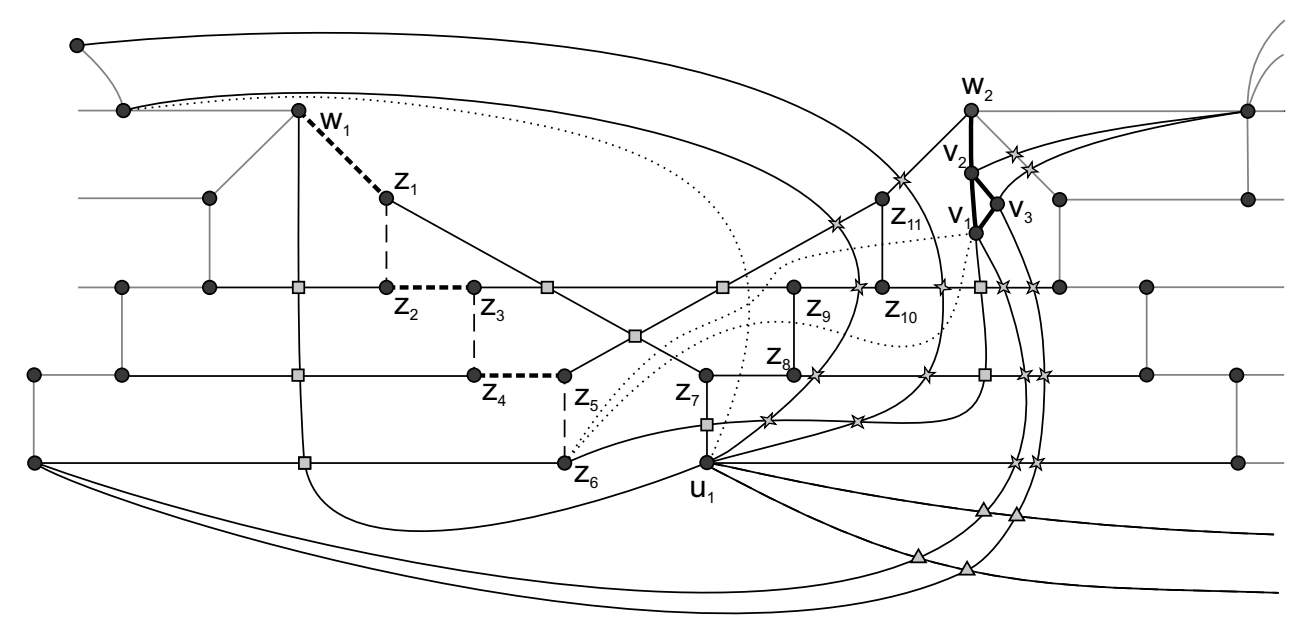

Figure 5: A fragment of an optimal tile drawing of $G_{2,5}{ }^{\ddagger}$. Grey triangles, 4-stars and squares present three types of crossings. For clearer presentation we use different $\ell$ compared to Figure 4. Here $\ell=2$ and $n=5$, which means that an optimal drawing has 29 crossings: $\ell^{2}=4$ of triangles, $2 \ell(n-1)=16$ of 4 -stars and $\left(\begin{array}{l}n \\ 2\end{array}\right)-1=9$ of squares. Dotted lines show other possible renderings for certain edges. Note that we only change a single edge in each alternative drawing. The same observation holds for the following two figures.

To show that each edge of $H_{\ell, n}$ is critical, we first present $H_{\ell, n}$ with 4 types of edges (see Figure 4): thin solid, thin dashed, thick dashed and thick solid. Then we present four figures, where each figure focuses its attention on one special type of edges: Figure 5 for thin solid edges, Figure 6 for thin dashed edges, Figure 7 for thick dashed edges and Figure 8 for thick solid edges. Every edge of a given type is crossed in the appropriate 
figure. Sometimes, some edge $e$ is used in different optimal drawings to cross different ones of the edges of the specific type. The required optimal redrawings of such edges $e$ are indicated with dotted curves.

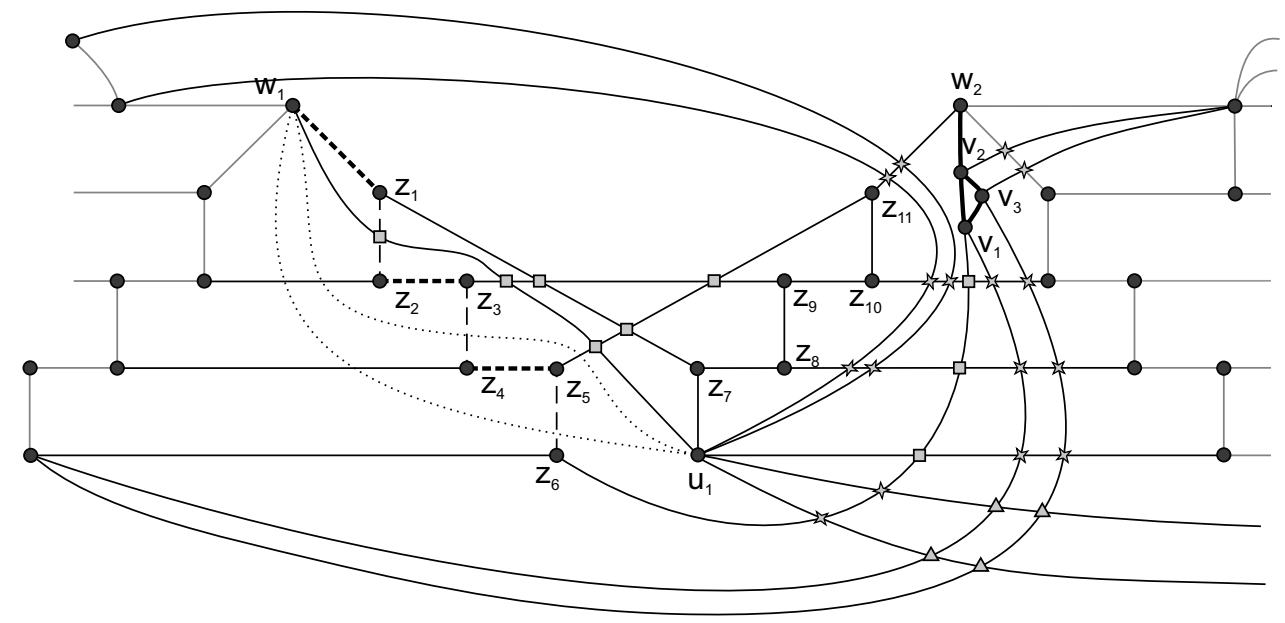

Figure 6: A fragment of an optimal tile drawing of $G_{2,5^{\uparrow}}$. See Figure 5 for additional explanation.

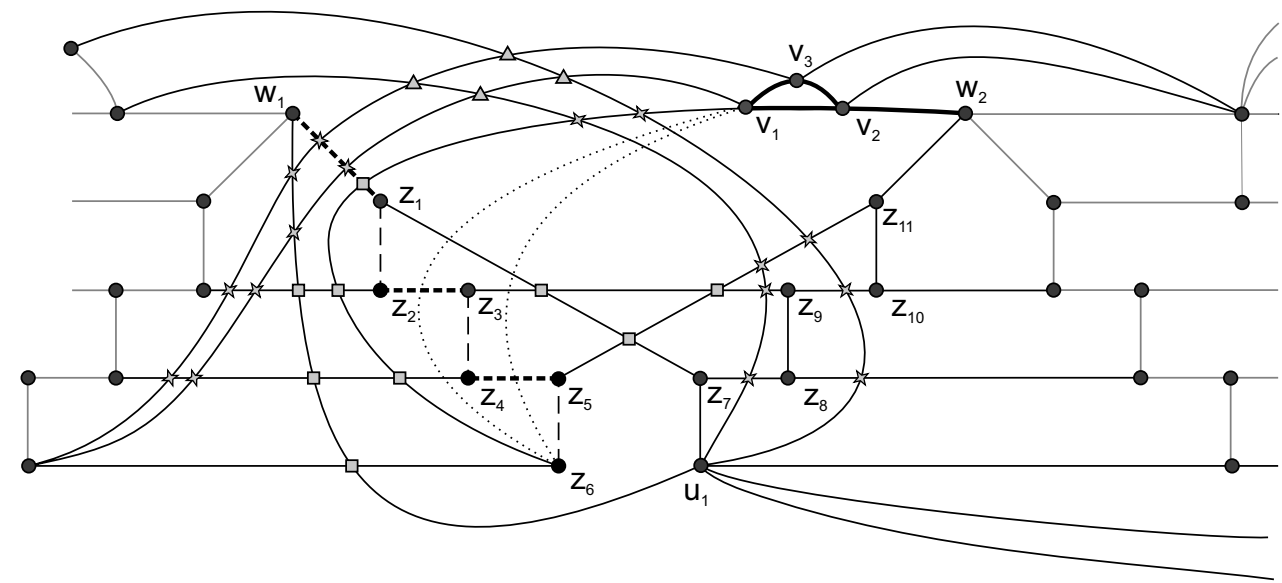

Figure 7: A fragment of an optimal tile drawing of $G_{2,5^{\uparrow}}$. See Figure 5 for additional explanation. 


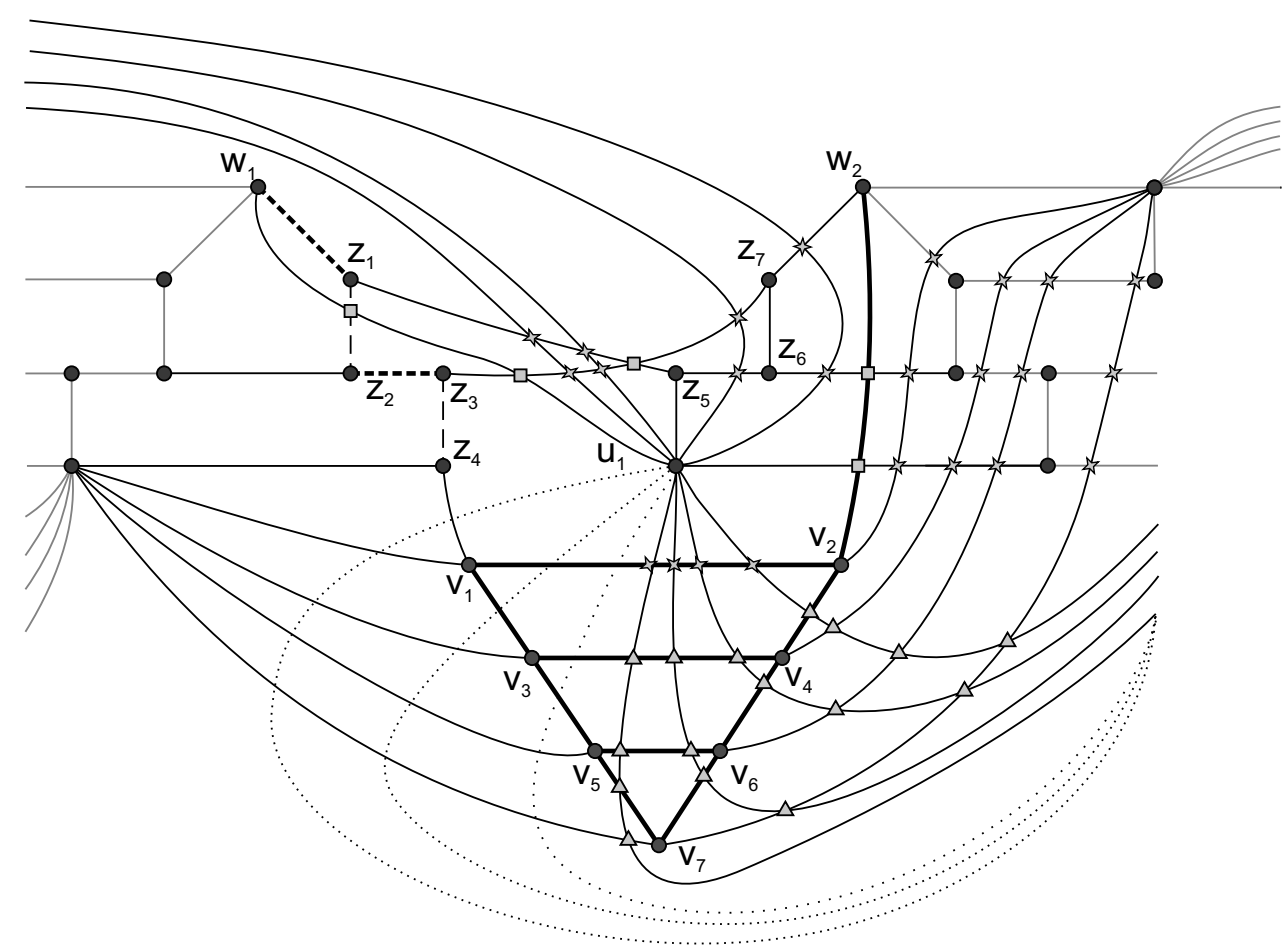

Figure 8: A fragment of an optimal tile drawing of $G_{4,4} \uparrow$. Here $\ell=n=4$, which means that an optimal drawing has 45 crossings: $\ell^{2}=16$ of triangles, $2 \ell(n-1)=24$ of 4 -stars and $\left(\begin{array}{l}n \\ 2\end{array}\right)-1=5$ of squares. For clearer presentation we use different $n$ compared to Figure 4. See Figure 5 for additional explanation.

\section{Families with Prescribed Frequent Degrees}

We now get back to the primary question which motivated the research leading to [11] and this paper: which vertex degrees other than 3,4,6 can occur arbitrarily often in infinite $k$-crossing-critical families? First, we summarize the relevant particular constructionsour future building blocks - obtained so far (note that some of the claimed results have been proved in a more general form than stated here, but we state them right in the form we shall use).

Proposition 4.1. There exist (infinite) families $\mathcal{F}$ of simple, 3 -connected, $k$-crossingcritical graphs such that, in addition, the following holds:

a) ([11, Section 4].) For every $k \geqslant 10$ or odd $k \geqslant 5$, and every rational $r \in\left(4,6-\frac{8}{k+1}\right)$, a family $\mathcal{F}$ which is $\{4,6\}$-max-universal and each member of $\mathcal{F}$ is of average degree exactly $r$, and another $\mathcal{F}$ which is $\{4\}$-max-universal and of average degree exactly 4. Every graph of the two families has the set of its vertex degrees equal to $\{3,4,6\}$ (e.g., degree 3 repeats six times in each). 
b) ([11, Section 3 and 4].) For every $\varepsilon>0$, any integer $k \geqslant 5$ and every set $D_{e}$ of even integers such that $\min \left(D_{e}\right)=4$ and $6 \leqslant \max \left(D_{e}\right) \leqslant 2 k-2$, a family $\mathcal{F}$ which is $D_{e}$-max-universal, and each graph of $\mathcal{F}$ has the set of its vertex degrees $D_{e} \cup\{3\}$ and is of average degree from the interval $(4,4+\varepsilon)$.

c) ([13] for $k=2$ and [3] for general $k$, see $G(0, n, m)$.) For every $k=\left(\begin{array}{l}n \\ 2\end{array}\right)-1$ where $n \geqslant 3$ is an integer, a family $\mathcal{F}$ which is $\{3,4\}$-max-universal and each member of $\mathcal{F}$ is of average degree equal to $3+\frac{1}{4 n-7}$.

d) $\left(G(\ell, 3, m)\right.$ in Theorem 3.2.) For every $k=\ell^{2}+4 \ell+2$ where $\ell \geqslant 1$ is an integer, a family $\mathcal{F}$ which is $\{3,4,2 \ell+3\}$-max-universal and each member of $\mathcal{F}$ is of average degree $5-\frac{4}{\ell+2}$.

Having the particular constructions of Proposition 4.1 and the zip product with Theorem 2.14 at hand, it is now quite easy to give the "ultimate" combined construction as follows. For two graph families $\mathcal{F}_{1}, \mathcal{F}_{2}$ of simple 2-connected graphs such that each graph in $\mathcal{F}_{1} \cup \mathcal{F}_{2}$ has a vertex of degree 3 , we define the zip product of $\mathcal{F}_{1}$ and $\mathcal{F}_{2}$ as the family of all graphs $H$ such that there exist $G_{1} \in \mathcal{F}_{1}, G_{2} \in \mathcal{F}_{2}$ and vertices $v_{1} \in V\left(G_{1}\right), v_{2} \in V\left(G_{2}\right)$ of degree 3 , and $H$ is the zip product of $G_{1}$ and $G_{2}$ according to $v_{1}, v_{2}$.

Lemma 4.2. Let $\mathcal{F}_{i}, i=1,2$, be a $D_{i}$-max-universal family of simple 2-connected graphs such that each graph in $\mathcal{F}_{i}$ has a vertex of degree 3 . Then the zip product of $\mathcal{F}_{1}$ and $\mathcal{F}_{2}$ is a $\left(D_{1} \cup D_{2}\right)$-max-universal family.

Proof. Let $\mathcal{F}$ denote the zip product of $\mathcal{F}_{1}$ and $\mathcal{F}_{2}$. We first prove that $\mathcal{F}$ is $\left(D_{1} \cup D_{2}\right)$ universal. Choose any set of integers $\left\{m_{d} \mid d \in D_{1} \cup D_{2}\right\}$, and, for $i=1,2$, graphs $G_{i} \in \mathcal{F}_{i}$, such that, for each $d \in D_{i} \backslash\{3\}, G_{i}$ contains at least $m_{d}$ vertices of degree $d$, and, if $3 \in D_{i}$, $G_{i}$ has at least $m_{3}+1$ vertices of degree 3 . Then, for each $d \in D_{1} \cup D_{2}$, the zip product of $G_{1}$ and $G_{2}$ (according to any pair of their degree-3 vertices) has at least $m_{d}$ vertices of degree $d$.

Conversely, assume that $\mathcal{F}$ is $\{d\}$-universal for some integer $d$. Then, for every integer $m$, there exists $G \in \mathcal{F}$ such that $G$ has at least $2 m$ vertices of degree $d$. Since $G$ is a zip product of graphs $G_{i} \in \mathcal{F}_{i}, i=1,2$, one of $G_{1}, G_{2}$ contains at least $m$ vertices of degree $d$. W.l.o.g., this happens infinitely often for $i=1$, and so (up to symmetry) $\mathcal{F}_{1}$ is $\{d\}$-universal. Therefore, $d \in D_{1} \cup D_{2}$ which proves that $\mathcal{F}$ is $\left(D_{1} \cup D_{2}\right)$-max-universal.

Theorem 4.3. Let $D$ be any finite set of integers such that $\min (D) \geqslant 3$. Then there is an integer $K=K(D)$, such that for every $k \geqslant K$, there exists a D-universal family of simple, 3 -connected, $k$-crossing-critical graphs. Moreover, if either $3,4 \in D$ or both $4 \in D$ and $D$ contains only even numbers, then there exists a D-max-universal such family. All the vertex degrees in the families are from $D \cup\{3,4,6\}$.

Proof. It suffices to prove the second claim ( $D$-max-universal) since a $(D \cup\{3,4\})$-maxuniversal family is also $D$-universal. Furthermore, if $D$ contains only even numbers, then the claim has already been proved in [11], here in Proposition 4.1 b). 
Hence assume the case $3,4 \in D$, and let $D_{e} \subseteq D$ be the subset of the even integers from $D$. Let $\mathcal{F}_{e}$ denote the family from Proposition $4.1 \mathrm{~b}$ ) with $k_{e}=\frac{1}{2} \max \left(D_{e}\right)+1$, and $\mathcal{F}_{3}$ the family from Proposition $4.1 \mathrm{c}$ ) with $k=2$. For every $a \in D \backslash D_{e}, a>3$, let $\mathcal{F}_{a}$ denote the family from Proposition $4.1 \mathrm{~d}$ ) with $2 \ell_{a}+3=a$ and crossing number $k_{a}=\ell_{a}^{2}+4 \ell_{a}+2$. Since, in particular, $\mathcal{F}_{3}$ is $\{3\}$-universal, we may assume that every graph in $\mathcal{F}_{3}$ has more than $\left|D \backslash D_{e}\right|$ vertices of degree 3 . We now construct a family $\mathcal{F}$ as the iterated zip product of $\mathcal{F}_{3}, \mathcal{F}_{e}$, and (possibly) of each $\mathcal{F}_{a}$ where $a \in D \backslash D_{e}, a>3$.

Clearly, every graph from $\mathcal{F}$ is simple and 3-connected. By Lemma $4.2, \mathcal{F}$ is $D$ max-universal, and by Theorem $2.14, \mathcal{F}$ is $K$-crossing-critical where $K=k_{e}+2+$ $\sum_{a \in D \backslash D_{e}, a>3} k_{a}$. This construction creates only vertices of degrees from $D \cup\{3,4,6\}$. To extend the construction of $\mathcal{F}$ to any parameter $k>K$, we simply replace the family $\mathcal{F}_{e}$ by analogous $\mathcal{F}_{e}^{\prime}$ from Proposition $\left.4.1 \mathrm{~b}\right)$ with the parameter $k_{e}^{\prime}=k_{e}+(k-K)$.

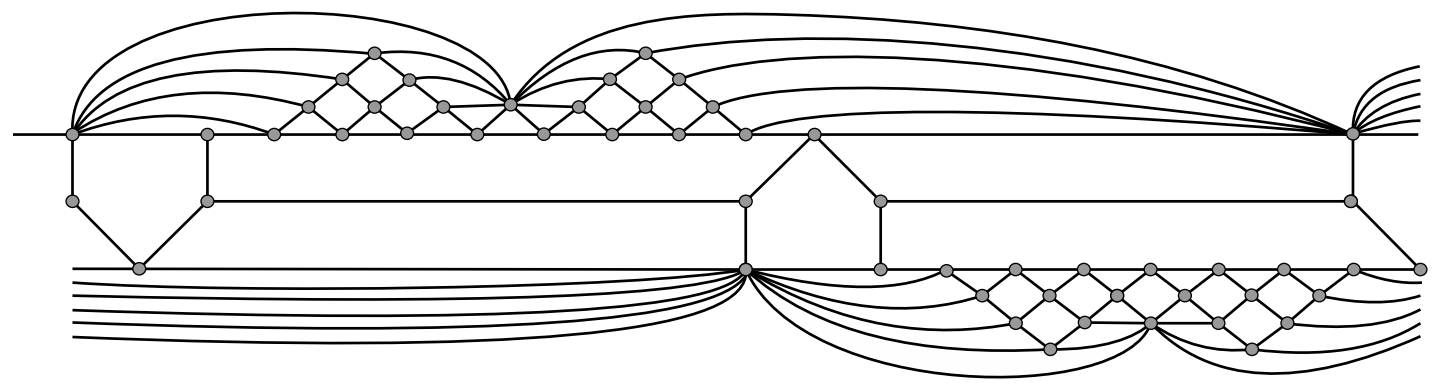

Figure 9: A possible (alternative) way of combining the ideas of the construction [11] with the tile $G_{5,3}$.

At last we shortly remark that building blocks of the "crossed belt" construction of [11] (Proposition $4.1 \mathrm{~b}$ ) can be directly combined with the new construction of $G(\ell, n, m)$, without invoking a zip product. Such a combination is outlined in Figure 9. However, since this construction can only achieve a combination of various even degrees with one prescribed odd degree (greater than 3), it cannot fully replace the proof of Theorem 4.3 and so we refrain from giving the lengthy technical details in this paper.

\section{Families with Prescribed Average Degree}

In addition to Theorem 4.3, we are going to show that the claimed $D$-max-universality property can be combined with nearly any feasible rational average degree of the family.

Theorem 5.1. Let $D$ be any finite set of integers such that $\min (D) \geqslant 3$ and $A \subset \mathbb{R}$ be an interval of reals. Assume that at least one of the following assumptions holds:

a) $D \supseteq\{3,4,6\}$ and $A=(3,6)$,

b) $D \supsetneq\{3,4\}$ and $A=(3,4]$, or $D=\{3,4\}$ and $A=(3,4)$, 
c) $D \supsetneq\{3,4\}$ and $A=\left(3,5-\frac{8}{b+1}\right)$ where $b$ is the largest odd number in $D$ and $b \geqslant 9$ (note that $b=7$ is covered in (b)),

d) $D \supseteq\{4,6\}$ contains only even numbers and $A=(4,6)$, or $D=\{4\}$ and $A=\{4\}$.

Then, for every rational $r \in A$, there is an integer $K=K(D, r)$ such that for every $k \geqslant K$, there exists a $D$-max-universal family of simple 3 -connected $k$-crossing-critical graphs of average degree precisely $r$.

Before we prove the theorem, we informally review the coming steps. The basic idea of balancing the average degree in a crossing-critical family is quite simple; assume we have two families $\mathcal{F}_{a}, \mathcal{F}_{b}$ of fixed average degrees $a<b$, respectively, and containing some degree-3 vertices. Then, we can use zip product of graphs from the two families to obtain new graphs of average degrees which are convex combinations of $a$ and $b$. This simple scheme, however, has two difficulties:

- If one combines graphs $G_{1} \in \mathcal{F}_{a}$ and $G_{2} \in \mathcal{F}_{b}$, then the average degree of the disjoint union $G_{1} \cup G_{2}$ is the average of $a, b$ weighted by the sizes of $G_{1}, G_{2}$. Hence we need great flexibility in choosing members of $\mathcal{F}_{a}, \mathcal{F}_{b}$ of various size, and this will be taken care of by the notion of a scalable family.

- Second, after applying a zip product of $G_{1}, G_{2}$ the resulting average degree is no longer this weighted average of $a, b$ but a slightly different rational number. We will take care of this problem by introducing a special compensation gadget whose role is to revert the change in average degree caused by the zip product.

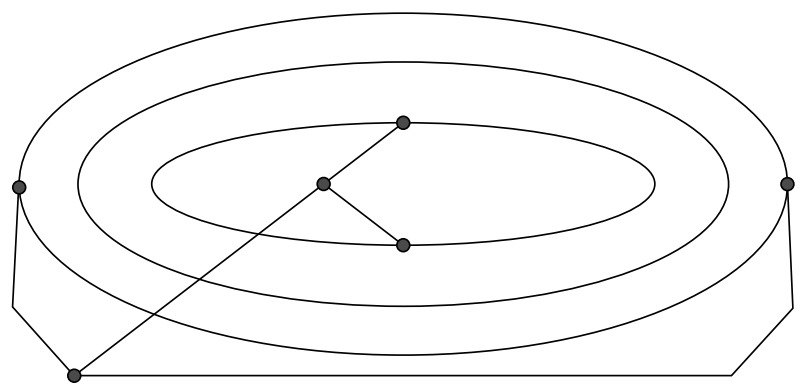

Figure 10: The $k$-crossing-critical "crossed belt" construction of [11]: the shaded part is any plane graph consisting of an edge-disjoint union of $k$ cycles, satisfying certain (rather weak) technical and connectivity conditions; the six marked vertices are all of degree three.

We start with addressing the second point. The compensation gadget (one for a whole family) will be picked from the family in Proposition 4.1 a). To describe it precisely, we have to (at least informally) introduce the very general crossed belt construction of crossing-critical families from [11] - see it is Figure 10. Let $T$ be the planar tile depicted in Figure 11 on the left, and let $M_{m}^{\prime}$ be the planar graph obtained as the cyclization 

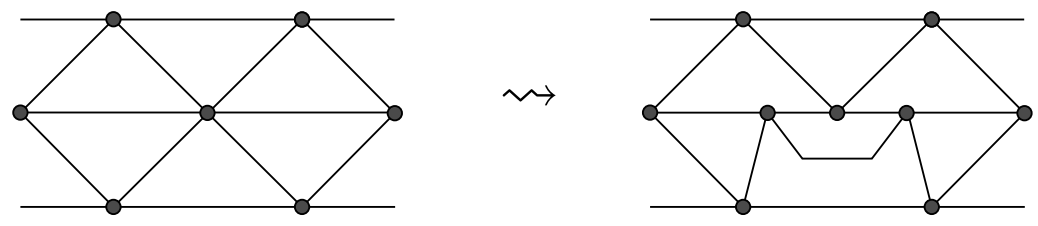

Figure 11: The tile $T$ (left) used to construct our "compensation gadget" $M_{m}$, and the tile $T^{\prime \prime}$ (called "double-split" in [11]) that can replace $T$ in the compensation gadget.

$\circ\left(T_{0}, \ldots, T_{m-1}\right)$ where each $T_{i}=T$. Let $M_{m}, m \geqslant 12$, be constructed from $M_{m}^{\prime}$ by adding six new degree-3 vertices and five new edges as in Figure 10, such that four of the new vertices subdivide rim edges of the tiles $T_{0}, T_{\lfloor m / 4\rfloor}, T_{\lfloor m / 2\rfloor}, T_{\lfloor 3 m / 4\rfloor}$. Let $M_{m}^{c}$ be constructed exactly as $M_{m}$ but replacing arbitrary $c \geqslant 0$ of the tiles $T$ with $T^{\prime \prime}$ shown on the right in Figure 11.

Proposition 5.2 ([11]). For any $m \geqslant 12$ and $0 \leqslant c \leqslant m$, the graph $M_{m}^{c}$ is 5 -crossingcritical.

The way "compensating by" the gadget $M_{m}^{c}$ works, is formulated next.

Lemma 5.3. Let $G_{1}, \ldots, G_{t}$ be graphs, each having at least two degree-3 vertices, and let $q \in \mathbb{N}$ and $m \geqslant \max (q+t, 12)$. If $H$ is a graph obtained using the zip product of all $G_{1}, \ldots, G_{t}$ and of $M_{m}^{q+t}$ (in any order and any way), then the average degree of $H$ is equal to the average degree of the disjoint union of $G_{1}, \ldots, G_{t}$ and $M_{m}^{q}$.

Proof. Let $n_{i}=\left|V\left(G_{i}\right)\right|$ and $s_{i}$ be the sum of degrees in $G_{i}$, and let $n_{0}=6 m+6+2 q$, $s_{0}=28 m+18+6 q$ be the same quantities in $M_{m}^{q}$. Then $n_{0}^{\prime \prime}=\left|V\left(M_{m}^{q+t}\right)\right|=n_{0}+2 t$ and the sum of degrees of $M_{m}^{q+t}$ is $s_{0}^{\prime \prime}=s_{0}+6 t$. Since performing one zip operation decreases the number of vertices by 2 and the sum of degrees by 6 , we have $|V(H)|=n_{0}^{\prime \prime}+n_{1}+\cdots+n_{t}-$ $2 t=n_{0}+n_{1}+\cdots+n_{t}$ and the sum of degrees in $H$ is $s_{0}^{\prime \prime}+s_{1}+\cdots+s_{t}-6 t=s_{0}+s_{1}+\cdots+s_{t}$, and the claim follows.

To address the first point, we give the following definition. A family of graphs $\mathcal{F}$ is scalable if all the graphs in $\mathcal{F}$ have equal average degree and for every $G \in \mathcal{F}$ and every integer $a$, there exists $H \in \mathcal{F}$ such that $|V(H)|=a|V(G)|$. Furthermore, $\mathcal{F}$ is $D$-maxuniversal scalable if, additionally, $H$ contains at least $a$ vertices of each degree from $D$ and the number of vertices of degrees not in $D$ is bounded from above independently of $a$.

Trivially, the families of Proposition $4.1 \mathrm{c}$ ), d) are $D$-max-universal scalable for $D=$ $\{3,4\}$ and $D=\{3,4,2 \ell+3\}$, respectively. For the families as in Proposition $4.1 \mathrm{a}), \mathrm{b})$, we have:

Lemma 5.4. There exist families, satisfying the conditions of Proposition 4.1 a) and b), respectively, which are D-max-universal scalable for their respective sets $D$.

Note that in the previous case of Proposition $4.1 \mathrm{~b}$ ), the claimed family from [11] was not required to have fixed average degree. Now also the family extending this case b) will be of fixed average degree. 
Proof. The proof is completely based on the constructions from [11], but since the question of scalability is not considered there, we have to discuss some further details of the crossed belt construction of [11] (recall Figure 10).

First, consider a $\{4\}$-max-universal family $\mathcal{F}_{4}$ of simple, 3 -connected, $k$-crossingcritical graphs of average degree 4 , as in Proposition 4.1 a). Pick any $G \in \mathcal{F}_{4}$; then $G$ has precisely six degree-3 vertices, and since the only other vertex degrees occurring in $G$ are 4 and $6, G$ has precisely three degree- 6 vertices. Let $G^{\prime}$ be the "planar belt" of $G$ (the shaded part in Figure 10, without degree-3 vertices). Then $G^{\prime}$ can be cut to form a perfect planar tile $T_{G^{\prime}}$ such that $\circ T_{G^{\prime}}=G^{\prime}$. For integer $a \geqslant 1$, let $G_{a}^{\prime}$ denote the cyclization of $a$ copies of $T_{G^{\prime}}$, and let $G_{a}^{\prime \prime}$ denote the graph $G_{a}^{\prime}$ with the six degree-3 vertices added back (such that four of them subdivide the same edges of one copy of the tile $T_{G^{\prime}}$ as they do in $G$ ). By [11], $G_{a}^{\prime \prime}$ is again $k$-crossing-critical. If $n=\left|V\left(G^{\prime}\right)\right|$ and $s$ is the degree sum of $G^{\prime}$, then $|V(G)|=n+6$ and the degree sum of $G$ is $s+18$. Furthermore, $\left|V\left(G_{a}^{\prime \prime}\right)\right|=a n+6$ and the degree sum of $G_{a}^{\prime \prime}$ is $a s+18$, and $G_{a}^{\prime \prime}$ has $3 a$ degree- 6 vertices. We denote by $G_{a}$ the graph obtained by $3 a-3$ "double split" operations each replacing a degree- 6 vertex by three degree- 4 vertices as illustrated in Figure 11. Then $\left|V\left(G_{a}\right)\right|=a n+6+2(3 a-3)=a|V(G)|$ and the degree sum of $G_{a}$ is $a s+18+6(3 a-3)=a(s+18)$, and so the average degree is the same as of $G$. There are only three degree- 6 vertices left in $G_{a}$. Hence, for every $a>1$, we may assume $G_{a} \in \mathcal{F}_{4}$ as well.

Second, consider a $\{4,6\}$-max-universal family $\mathcal{F}_{r}$ of simple 3 -connected $k$-crossingcritical graphs of average degree $r \in\left(4,6-\frac{8}{k+1}\right)$, as in Proposition 4.1 a). Then the proof follows the same line as in the previous paragraph, only that now we have many degree- 6 vertices by the assumption of $\{6\}$-universality.

Third, consider a $D_{e}$-max-universal family $\mathcal{F}_{e}$ of simple 3 -connected $k$-crossing-critical graphs, as in Proposition $4.1 \mathrm{~b}$ ). This case is somehow different from the previous two since we have no vertices of degree 6 (unless $6 \in D_{e}$ ) and $\mathcal{F}_{e}$ contains graphs of various average degrees. Though, for any fixed $\varepsilon>0, \mathcal{F}_{e}$ can be chosen such that the average degree of every member of $\mathcal{F}_{e}$ is from the interval $(4,4+\varepsilon / 2)$. Pick arbitrary but sufficiently large $G \in \mathcal{F}_{e}$. Then one can find (see [11] for details) three edges in $G$ not close to each other and not having vertices of degree other than 4 in close neighbourhood, and let $G_{1}$ be obtained by contracting these three edges (into vertices of degree 6). By [11], $G_{1}$ is again $k$-crossing-critical. Since $G$ is sufficiently large, the average degree of $G_{1}$ is equal to some $r_{1} \in(4,4+\varepsilon)$. Now the construction from the first case above applies to $G_{1}$ and gives a whole scalable family of average degree $r_{1}$.

The next step is to combine suitable scalable families to obtain arbitrary rational average degrees in a given interval (roughly, between the sparsest and the densest available family).

Lemma 5.5. Assume, for $i=1, \ldots, t$, that $\mathcal{F}_{i}$ is a $D_{i}$-max-universal scalable family of simple 3-connected $k_{i}$-crossing-critical graphs of average degree exactly $r_{i}$, and that every graph in $\mathcal{F}_{1} \cup \cdots \cup \mathcal{F}_{t}$ has at least two degree-3 vertices. For every $k \geqslant k_{1}+\cdots+k_{t}+5$, there exists rational $r_{0} \in(3,6)$, such that the following holds for every $a_{1}, \ldots, a_{t}, c \in \mathbb{N}$ : 
a) there exists a simple, 3 -connected, $k$-crossing-critical graph $G$ having at least $a_{i}$ vertices of each degree from $D_{i}$,

b) the number of vertices of $G$ of degree not in $D_{1} \cup \cdots \cup D_{t}$ is bounded from above by a number depending only on $c, k$ and the families $\mathcal{F}_{1}, \ldots, \mathcal{F}_{t}$, and

c) the average degree of $G$ is precisely

$$
r=\frac{\sum_{i=1}^{t} a_{i} r_{i}+c r_{0}}{\sum_{i=1}^{t} a_{i}+c} .
$$

Proof. Let $\ell=k-\left(k_{1}+\cdots+k_{t}+5\right)$ and denote by $\mathcal{K}_{\ell}$ a set of $\ell$ disjoint copies of the graph $K_{3,3}$. Pick arbitrary $G_{i} \in \mathcal{F}_{i}, i=1, \ldots, t$. We may w.l.o.g. assume that $n_{0}=\left|V\left(G_{1}\right)\right|=\cdots=\left|V\left(G_{t}\right)\right|$ and $n_{0}$ divisible by 6 , since otherwise we take the least common multiple of 6 and all the graph sizes and apply scalability of the families $\mathcal{F}_{i}$. Clearly, $n_{0}$ can be chosen arbitrarily large as well, such as $n_{0} \geqslant 6(4 \ell+t+4)$. Let $G_{0}=M_{n_{0} / 6-(\ell+1)}^{0}$ (the compensation gadget defined above) and $H_{0}$ denote the disjoint union of $\mathcal{K}_{\ell}$ and $G_{0}$. Then $\left|V\left(H_{0}\right)\right|=n_{0}$ and we choose $r_{0}$ to be the average degree of $H_{0}$;

$$
r_{0}=\frac{18 \ell+28\left(\frac{n_{0}}{6}-(\ell+1)\right)+18}{n_{0}}=\frac{\frac{14 n_{0}}{3}-10(\ell+1)}{n_{0}} .
$$

Again by scalability, for $i=1, \ldots, t$, there exist $G_{i}^{* a_{i}} \in \mathcal{F}_{i}$ (of average degree $r_{i}$ ) such that $\left|V\left(G_{i}^{* a_{i}}\right)\right|=a_{i} n_{0}$. Similarly, we let $G_{0}^{* c}=M_{c n_{0} / 6-c(\ell+1)}^{3(c-1)(\ell+1)}$. It is simple calculus to verify that the disjoint union of $\mathcal{K}_{\ell}$ and $G_{0}^{* c}$ has $c n_{0}$ vertices and the average degree equal to

$$
\frac{18 \ell+28\left(\frac{c n_{0}}{6}-c(\ell+1)\right)+18(c-1)(\ell+1)+18}{c n_{0}}=\frac{\frac{14 c n_{0}}{3}-10 c(\ell+1)}{c n_{0}}=r_{0} .
$$

Hence the average degree of the disjoint union of $\mathcal{K}_{\ell}$ and $G_{0}^{* c}$ and $G_{1}^{* a_{1}}, \ldots, G_{t}^{* a_{t}}$ indeed is

$$
\frac{\sum_{i=1}^{t} a_{i} n_{0} r_{i}+c n_{0} r_{0}}{\sum_{i=1}^{t} a_{i} n_{0}+c n_{0}}=r .
$$

Finally, we let $G_{0}^{\prime * c}=M_{c n_{0} / 6-c(\ell+1)}^{3(c-1)(\ell+1)+\ell+t}$ and construct the simple 3-connected graph $G$ as the zip product of $\mathcal{K}_{\ell}$ and $G_{0}^{* c}$ and $G_{1}^{* a_{1}}, \ldots, G_{t}^{* a_{t}}$. By Theorem 2.14, $G$ is $k$-crossingcritical with $k=\ell+5+k_{1}+\cdots+k_{t}$, as required. The degrees condition in a) follows from max-universal scalability of $\mathcal{F}_{1}, \ldots, \mathcal{F}_{t}$, and b) then follows as well since the size of $G_{0}^{\prime * c}$ is bounded with respect to $c, k$. Moreover, by compensation Lemma 5.3 , the average degree of $G$ is equal to $r$, as in (2).

Corollary 5.6 (Lemma 5.5). Assume $D_{i}$-max-universal scalable $k_{i}$-crossing-critical families $\mathcal{F}_{i}$ of average degree $r_{i}, i=1, \ldots, t$, as in Lemma 5.5, such that $r_{1}<r_{2}$. Then, for every $k \geqslant k_{1}+\cdots+k_{t}+5$ and every $r \in\left(r_{1}, r_{2}\right) \cap \mathbb{Q}$, there exists a $\left(D_{1} \cup \cdots \cup D_{t}\right)$ max-universal family of simple, 3 -connected, $k$-crossing-critical graphs of average degree exactly $r$. 
Proof. The proof is a simple exercise in calculus based on Lemma 5.5. Let $r=\frac{p}{q}$ where $p, q$ are relatively prime integers. Our task is to find infinitely many suitable choices of $a_{1}, \ldots, a_{t}$ such that, by $(1)$,

$$
\frac{p}{q}=\frac{\sum_{i=1}^{t} a_{i} r_{i}+c r_{0}}{\sum_{i=1}^{t} a_{i}+c}
$$

for some (unknown) rational $r_{0} \in(3,6)$ and suitable (but fixed, see below) $c$.

To further simplify the task, we choose sufficiently large integer $m$ such that $r_{1}^{\prime}=$ $\left(m r_{1}+r_{3}+\cdots+r_{t}\right) /(m+t-2)<r$ and set $a_{1}=m a, a_{3}=\cdots=a_{t}=a, a_{2}=b$ for yet unknown $a, b$. Then (3) reads:

$$
\frac{p}{q}=\frac{m a r_{1}+a r_{3}+\cdots+a r_{t}+b r_{2}+c r_{0}}{a(m+t-2)+b+c}=\frac{a(m+t-2) r_{1}^{\prime}+b r_{2}+c r_{0}}{a(m+t-2)+b+c}
$$

Let $s=m+t-2$, and $r_{1}^{\prime}=\frac{p_{a}}{q_{a}}, r_{2}=\frac{p_{b}}{q_{b}}, r_{0}=\frac{p_{0}}{q_{0}}$. We continue with equivalent processing:

$$
\begin{gathered}
\frac{p}{q}=\frac{a s \frac{p_{a}}{q_{a}}+b \frac{p_{b}}{q_{b}}+c \frac{p_{0}}{q_{0}}}{a s+b+c} \\
p(a s+b+c) q_{a} q_{b} q_{0}=a s q q_{b} q_{0} p_{a}+b q q_{a} q_{0} p_{b}+c q q_{a} q_{b} p_{0}
\end{gathered}
$$

Finally, we get that (3) under our special substitution for $a_{1}, \ldots, a_{t}$, is equivalent to the following linear Diophantine equation in $a, b$ :

$$
a \cdot s q_{b} q_{0}\left(p q_{a}-p_{a} q\right)+b \cdot q_{a} q_{0}\left(p q_{b}-p_{b} q\right)=c q_{a} q_{b}\left(p_{0} q-p q_{0}\right)
$$

Setting $c=q_{0} \cdot G C D\left(s q_{b}\left(p q_{a}-p_{a} q\right), q_{a}\left(p q_{b}-p_{b} q\right)\right)$, this equation has infinitely many integer solutions, and since $r_{1}^{\prime}<r<r_{2}$, we have that $p q_{a}-p_{a} q>0$ and $p q_{b}-p_{b} q<0$ and so infinitely many of the solutions are among positive integers (regardless of whether the right-hand side is positive, zero or negative).

Proof of Theorem 5.1. The case d) has already been proved in [11], see Proposition $4.1 \mathrm{a}$ ). In all other cases, let $\mathcal{F}_{1}$ be the family from Proposition $4.1 \mathrm{c}$ ) such that the parameter $n$ satisfies $r_{1}=3+\frac{1}{4 n-7}<r$ (where $r \in A \cap \mathbb{Q}, r>3$, is the desired fixed average degree).

In the case a), let $\mathcal{F}_{2}$ be a family from Proposition 4.1 a) with average degree equal to arbitrary (but fixed) $r_{2} \in(r, 6) \neq \varnothing$, which can be chosen as scalable by Lemma 5.4. In the case $\mathrm{c}$ ), let $\mathcal{F}_{2}$ be the family from Proposition $4.1 \mathrm{~d}$ ) with the parameter $\ell$ such that $b=2 \ell+3$; in this case $r_{2}=5-\frac{8}{b+1}>r$. Finally, we consider the remaining subcases of b). If $D=\{3,4\}$, then let $\mathcal{F}_{2}$ be the second family from Proposition 4.1 a) with average degree $r_{2}=4$. If $D \supsetneq\{3,4\}$, then let $\mathcal{F}_{2}$ be the family from Proposition $4.1 \mathrm{~b}$ ), made scalable and of fixed average degree $r_{2}>4$ by Lemma 5.4 .

In each one of the choices of $\mathcal{F}_{1}, \mathcal{F}_{2}$ above, $r_{1}<r<r_{2}$ holds. Furthermore, if necessary in order to fulfil $D$-max-universality, we introduce additional scalable families $\mathcal{F}_{3}, \ldots$ as in the proof of Theorem 4.3. Then, Theorem 5.1 follows directly from Corollary 5.6. 


\section{Degree Properties in 2-Crossing-Critical Families}

In the previous constructions, we have always assumed that the fixed crossing number $k$ of the families is sufficiently large. One can, on the other hand, ask what happens if we fix a (small) value of $k$ beforehand (i.e., independently of the asked degree properties).

In this direction, there is the remarkable result of Dvořák and Mohar [6] proving the existence of $k$-crossing-critical families with unbounded maximum degree for any $k \geqslant 171$. Unfortunately, since [6] is not really constructive, we do not know anything exact about the degrees occurring in these families. An explicit construction of a $k$-crossing-critical family with unbounded maximum degree is known only in the projective plane [12] for $k \geqslant 2$, but that falls outside of the area of interest of this paper.
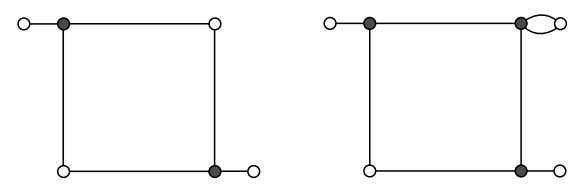

Figure 12: The two frames.
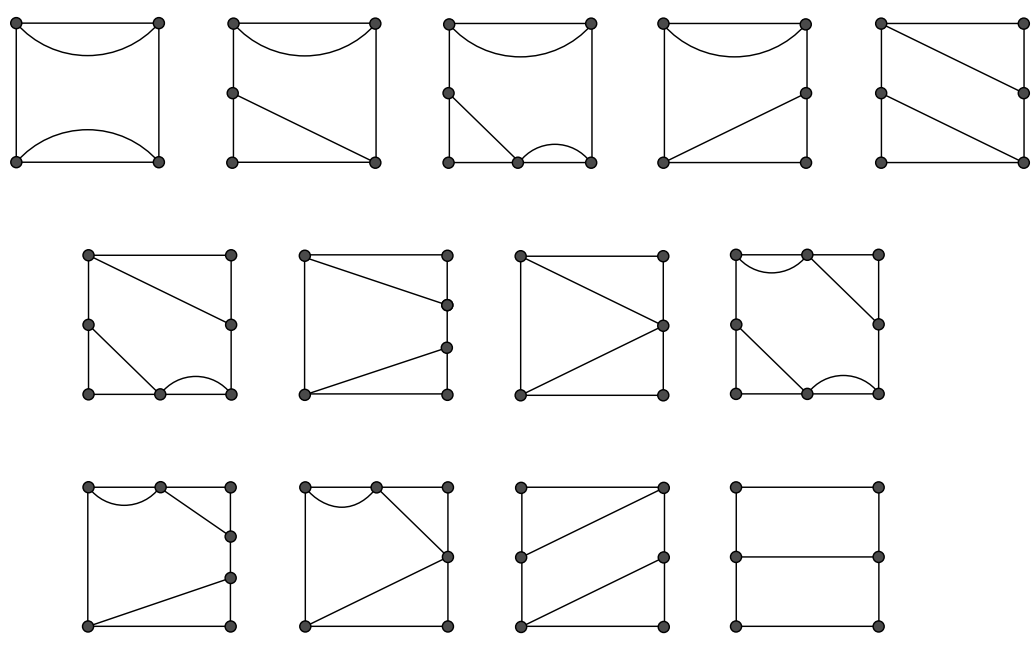

Figure 13: The thirteen pictures for Definition 6.1.

It thus appears natural to thoroughly investigate the least non-trivial case of $k=2$, with significant help of the characterization result [5]. In a nutshell, [5] claims that nearly all 2-crossing-critical graphs are built from a certain rather small finite set of tiles. The formal result is stated next.

We refer to Section 2.3 for definitions of the operations $\circ, \otimes$ and $\downarrow$ on tiles.

Definition 6.1. Let $\mathcal{S}$ denote the set of tiles which are obtained as combinations of one of the two frames, illustrated in Figure 12, and the 13 pictures, shown in Figure 13. 


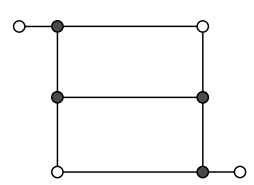

(a) tile $T_{a}$

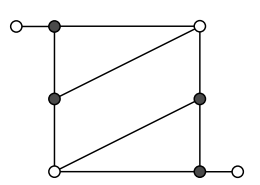

(b) tile $T_{b}$

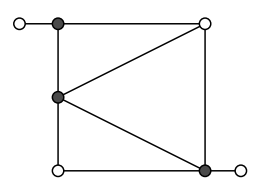

(c) tile $T_{c}$

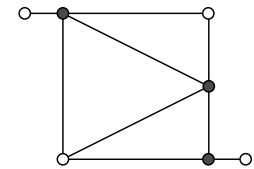

(d) tile $T_{d}$

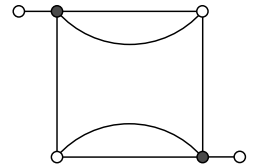

(e) tile $T_{e}$

Figure 14: Examples of tiles from $\mathcal{S}$.

These are combined in a way that a picture is inserted into a frame by identifying the two geometric squares (typically by subdividing some edges of the frame). A given picture may be inserted into a frame either with the given orientation or with a $180^{\circ}$ rotation. Note that $\mathcal{S}$ contains 42 different tiles.

We inductively define the following: The set of odd tiles $\mathcal{T}_{o}(\mathcal{S})$ consists of all the tiles that are either in $\mathcal{S}$ or are obtained as $T_{e} \otimes T$ with $T_{e} \in \mathcal{T}_{e}$ and $T \in \mathcal{S}$. The set of even tiles $\mathcal{T}_{e}(\mathcal{S})$ consists precisely of the tiles obtained as $T_{o} \oplus^{\uparrow} T^{\uparrow}$, where $T_{o} \in \mathcal{T}_{o}(\mathcal{S})$ and $T \in \mathcal{S}$. Note that an odd number of tiles of $\mathcal{S}$ is used to construct a tile of $\mathcal{T}_{o}(\mathcal{S})$, and an even number for $\mathcal{T}_{e}(\mathcal{S})$.

Let the set $\mathcal{G}(\mathcal{S})$ consist precisely of all the graphs of the form $G=\circ(T)$ where $T \in$ $\mathcal{T}_{o}(\mathcal{S}) \backslash \mathcal{S}$. Note that each graph of $\mathcal{G}(\mathcal{S})$ is obtained as $G=\circ\left(\mathcal{T}^{\downarrow}\right)$, where $\mathcal{T}$ is a sequence $\left(T_{0},{ }^{\uparrow} T_{1}{ }^{\uparrow}, T_{2}, \ldots,{ }^{\uparrow} T_{2 m-1}{ }^{\uparrow}, T_{2 m}\right)$ such that $m \geqslant 1$ and $T_{i} \in \mathcal{S}$ for each $i=0,1,2, \ldots, 2 m$.

Some examples of tiles from $\mathcal{S}$, hereafter named from $T_{a}$ to $T_{e}$, are shown in Figure 14. We will use these tiles from $T_{a}$ to $T_{e}$ in what follows.

Theorem 6.2. ([5]) There exist only finitely many 3-connected 2-crossing-critical graphs which do not contain a subdivision of the graph $V_{10}$, which is obtained from a 10-cycle by adding all the 5 main diagonals.

Then, $G$ is a 3-connected 2-crossing-critical graph containing a subdivision of $V_{10}$, if and only if $G \in \mathcal{G}(\mathcal{S})$.

Since we are interested exclusively in infinite families of 2-crossing-critical graphs, we can focus on the graphs in $\mathcal{G}(\mathcal{S})$, as any remaining (necessarily finite) subset of graphs disjoint from $\mathcal{G}(\mathcal{S})$ would not affect degree properties of our families. Note also that any 3-connected, 2-crossing-critical family of graphs contains at most finitely many graphs which are not almost-planar because any graph from $\mathcal{G}(\mathcal{S})$ is almost-planar.

Theorem 6.3. A 3-connected 2-crossing-critical D-max-universal family

a) of simple graphs exists if and only if $\{3\} \subsetneq D \subseteq\{3,4,5,6\}$.

b) exists if and only if $D \subseteq\{3,4,5,6\},|D| \geqslant 2$, and $D \cap\{3,4\} \neq \varnothing$. 


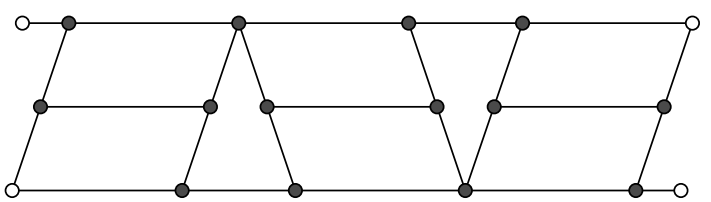

(a) $\otimes\left(T_{a},{ }^{\uparrow} T_{a}{ }^{\uparrow}, T_{a}\right)$ for $\{3,4\}$.

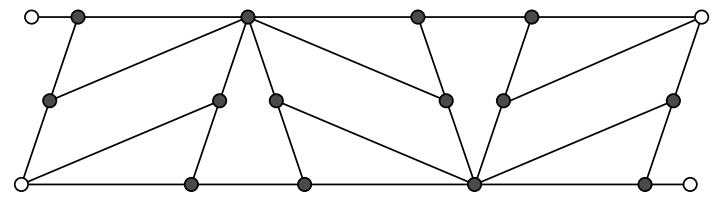

(c) $\otimes\left(T_{b},{ }^{\uparrow} T_{b} \uparrow, T_{b}\right)$ for $\{3,6\}$.

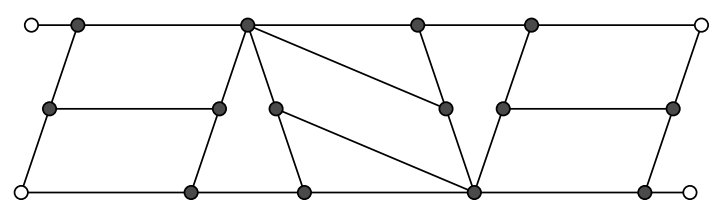

(b) $\otimes\left(T_{a},{ }^{\uparrow} T_{b} \uparrow, T_{a}\right)$ for $\{3,5\}$.

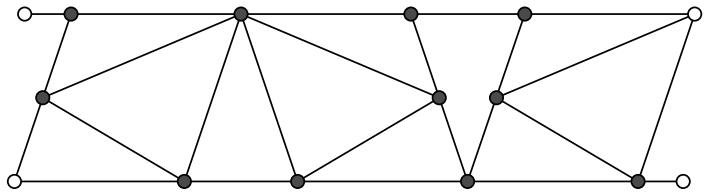

(d) $\otimes\left(T_{c},{ }^{\uparrow} T_{d}^{\ddagger}, T_{c}\right)$ for $\{3,4,6\}$.

Figure 15: Tiles which can be used to construct the family $\mathcal{F}$ in the proof of Theorem $6.3 \mathrm{a}$ ) in four particular cases of $D$. The remaining constructions can be obtained, e.g., as follows: the base tile for $\{3,4,5\}$ is a join of two tiles (a) and one tile (b), for $\{3,5,6\}$ it is a join of two tiles (b) and one tile (c), and for $\{3,4,5,6\}$ it is a join of two tiles (b) and one tile (d).

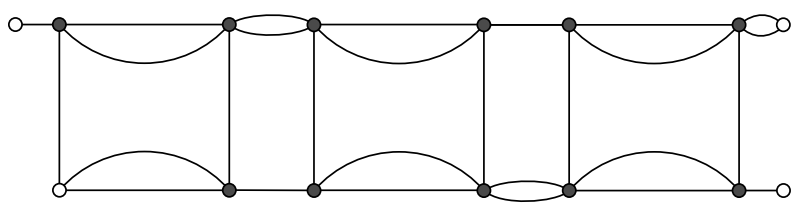

(a) $\otimes\left(T_{f}, \uparrow T_{f} \uparrow, T_{f}\right)$ for $\{4,5\}$.

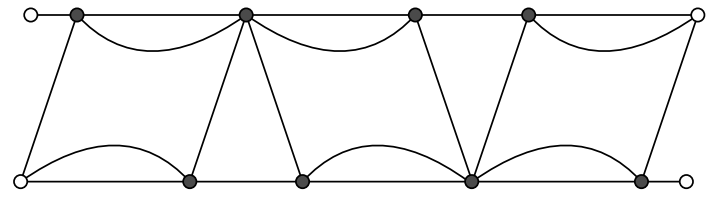

(b) $\otimes\left(T_{e},{ }^{\uparrow} T_{e}^{\uparrow}, T_{e}\right)$ for $\{4,6\}$.

Figure 16: Two non-simple tiles which cover cases $D \cap\{3,4\}=\{4\}$ for Theorem $6.3 \mathrm{~b}$ ). The set $\{4,5,6\}$ can be obtained as a combination of these two tiles.

Proof. Let $D$ be a set of positive integers and let $\mathcal{F}$ be any 3-connected 2-crossing-critical $D$-max-universal family. By Theorem 6.2, we may assume $\mathcal{F} \subseteq \mathcal{G}(\mathcal{S})$. For case a), there are only nine simple tiles in $\mathcal{S}$, and by join of any two of them we can only construct vertices with degrees $3,4,5$ and 6 , so $D \subseteq\{3,4,5,6\}$. On the other hand, any simple tile from $\mathcal{S}$ has a vertex of degree 3 that is not in its left or right wall, so $\{3\} \subseteq D$, and we get some other vertex with degree not equal to 3 after we join any two of them, so $\{3\} \subsetneq D$.

Now it only remains to construct a family $\mathcal{F}$ for a set $D$ such that $\{3\} \subsetneq D \subseteq$ $\{3,4,5,6\}$. For that see pictures in Figure 15 .

We do similar for case b). Using Theorem $6.3 \mathrm{a}$ ) (for $D$ such that $3 \in D$ ), only the cases $D \cap\{3,4\}=\{4\}$ remain to be resolved. For that see pictures of constructions in Figure 16.

In order to study average degrees in 2-crossing-critical graphs, we introduce the density characteristics of a tile $T$ as the pair of integers $(a, b)$, where (i) $a$ is the number of vertices of $T$, counting wall vertices of degree greater than 1 as $1 / 2$ and those of degree 1 as 0 , and (ii) $b$ is the sum of degrees of $T$ counting all degrees in full except those of wall vertices 
with degree 1 . Then we define the density of a tile $T$ as $\frac{b}{a}$. Relevance of this concept is revealed through the following lemma:

Lemma 6.4. Let $T \in \mathcal{T}_{o}(\mathcal{S})$ be the join of $\mathcal{T}=\left(T_{0},{ }^{\uparrow} T_{1} \uparrow, T_{2}, \ldots,{ }^{\uparrow} T_{2 m-1}{ }^{\uparrow}, T_{2 m}\right)$ so that $m \geqslant 1$ and $T_{i} \in \mathcal{S}$ for each $i=0,1,2, \ldots, 2 m$, let $G=\circ\left(\mathcal{T}^{\downarrow}\right)$, and let $\left(a_{i}, b_{i}\right)$ be the density characteristics of the tile $T_{i}$. Then the average degree of $G$ is equal to $\left(\sum_{i=0}^{2 m} b_{i}\right) /\left(\sum_{i=0}^{2 m} a_{i}\right)$. Hence if each $T_{i}$ has density $\frac{b}{a}$, then the average degree of $G$ is equal to their density.

Lemma 6.4 implies that the lowest and highest achievable average degrees in an infinite family of 2-crossing-critical graphs are determined by the lowest and highest density of some tiles in $S$. This implies the following theorem:

Theorem 6.5. A simple, 3-connected, 2-crossing-critical infinite family of graphs with average degree $r \in \mathbb{Q}$ exists if and only if $r \in\left[3 \frac{1}{5}, 4\right]$.

Proof. An elementary checking yields that the smallest density of a simple tile in $T$ is $3 \frac{1}{5}$, achieved by $T_{a}$ of characteristics $(5,16)$, and the largest is 4 , achieved by $T_{c}$ and $T_{d}$ of characteristics $(4,16)$. Lemma 6.4 combined with Theorem 6.2 implies that the average degree $r$ of an infinite family must be in the specified interval. Sequences consisting of just one of these tiles establish the boundary cases $r \in\left\{3 \frac{1}{5}, 4\right\}$, which are thus easily achievable.

Let $r=\frac{p}{q} \in\left(3 \frac{1}{5}, 4\right)$. Since sequences consisting of only $T_{a}$ and $T_{c}, T_{d}$ may violate the parity condition trying to establish average degree $r$, we add also some tiles $T_{b}$ with characteristics $(5,18)$ to the construction. Solving diophantine equations, we find a solution

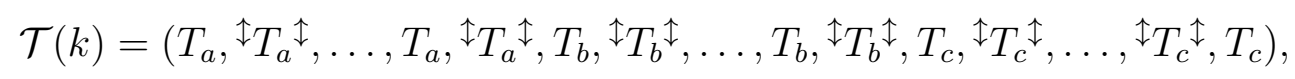

where tiles $T_{a}$ and ${ }^{\uparrow} T_{a} \uparrow$ together appear $\ell_{k}=(96 q-24 p-4)(2 k-1)$-times, $T_{b}$ and ${ }^{\mathfrak{}} T_{b}{ }^{\uparrow}$ together appear $m_{k}=8(2 k-1)$-times, and $T_{c},{ }^{\uparrow} T_{c}{ }^{\uparrow}$ together appear $n_{k}=(30 p-96 q-$ $5)(2 k-1)$-times.

Since $3 \frac{1}{5}<r<4$, we have $\ell_{k}, n_{k}>0$ for sufficiently large $p, q$ (which are not required to be relatively prime) such that $r=\frac{p}{q}$. The total length of the sequence $\mathcal{T}(k)$ then is $(6 p-1)(2 k-1)$, which is an odd number required for $\circ \mathcal{T}(k)^{\uparrow} \in \mathcal{G}(\mathcal{S})$. Now Lemma 6.4 and a routine calculation imply that $\mathcal{F}_{r}=\left\{\circ \mathcal{T}(k)^{\mathfrak{}} \mid k \in \mathbb{N}\right\}$ is a family of simple, 3-connected, 2-crossing-critical graphs with average degree $\frac{96 p(2 k-1)}{96 q(2 k-1)}=\frac{p}{q}=r$.

Theorem 6.6. A 3-connected, 2-crossing-critical infinite family with average degree $r \in \mathbb{Q}$ exists if and only if $r \in\left[3 \frac{1}{5}, 4 \frac{2}{3}\right]$.

Proof. The argument is as in the previous proof, with an additional observation that the largest possible density $4 \frac{2}{3}$ among non-simple tiles in $\mathcal{S}$ is obtained by the tile $T_{e}$ of characteristics $(3,14)$. For $r=\frac{p}{q} \in\left(3 \frac{1}{5}, 4 \frac{2}{3}\right)$, we use the sequence

$$
\mathcal{T}(k)=\left(T_{a},{ }^{\uparrow} T_{a}{ }^{\uparrow}, \ldots, T_{a},{ }^{\mathfrak{}} T_{a}{ }^{\uparrow}, T_{c},{ }^{\mathfrak{}} T_{c}{ }^{\uparrow}, \ldots,{ }^{\mathfrak{}} T_{c}{ }^{\uparrow}, T_{c},{ }^{\mathfrak{}} T_{e}{ }^{\uparrow}, T_{e}, \ldots,{ }^{\mathfrak{}} T_{e}{ }^{\uparrow}, T_{e}\right),
$$


where $T_{a}$ and ${ }^{\uparrow} T_{a}{ }^{\downarrow}$ together appear $(112 q-24 p-4)(2 k-1)$-times, $T_{c}$ and ${ }^{\uparrow} T_{c}{ }^{\uparrow}$ together appear $11(2 k-1)$-times, and $T_{e}$ and ${ }^{\uparrow} T_{e}^{\uparrow}$ together appear $(40 p-128 q-8)(2 k-1)$-times. The length of this sequence is $(16 p-16 q-1)(2 k-1)$ which is an odd number, and the average degree of each graph $\circ\left(\mathcal{T}(k)^{\uparrow}\right)$ is $r$.

We finish with the last theorem which summarizes results of this and previous section.

Theorem 6.7. Let $D$ be such that there exists a D-max-universal 3-connected 2-crossingcritical family. Then let $I_{D}$ (or $I_{D}^{s}$ for simple graphs) be the set of all rational numbers, such that there is a D-max-universal 3-connected 2-crossing-critical (simple) family with average degree $r$ if and only if $r \in I_{D}\left(r \in I_{D}^{s}\right)$. Then $I_{D}^{s}$ and $I_{D}$ are rational intervals and moreover:

\begin{tabular}{|c||c|c|}
\hline$D$ & $I_{D}^{s}$ & $I_{D}$ \\
\hline \hline$\{3,4\}$ & {$\left[\frac{16}{5}, \frac{18}{5}\right]$} & {$\left[\frac{16}{5}, \frac{15}{4}\right]$} \\
\hline$\{3,5\}$ & $\left\{\frac{17}{5}\right\}$ & {$\left[\frac{17}{5}, \frac{11}{3}\right]$} \\
\hline$\{3,6\}$ & $\left\{\frac{18}{5}\right\}$ & $\left\{\frac{18}{5}\right\}$ \\
\hline$\{4,5\}$ & $\varnothing$ & $\left\{\frac{9}{2}\right\}$ \\
\hline$\{4,6\}$ & $\varnothing$ & $\left\{\frac{14}{3}\right\}$ \\
\hline$\{3,4,5\}$ & $\left(\frac{16}{5}, 4\right]$ & $\left(\frac{16}{5}, \frac{9}{2}\right)$ \\
\hline$\{3,4,6\}$ & $\left(\frac{16}{5}, 4\right]$ & $\left(\frac{16}{5}, \frac{14}{3}\right)$ \\
\hline$\{3,5,6\}$ & $\left(\frac{17}{5}, \frac{18}{5}\right)$ & $\left(\frac{17}{5}, \frac{11}{3}\right)$ \\
\hline$\{4,5,6\}$ & $\varnothing$ & $\left(\frac{9}{2}, \frac{14}{3}\right)$ \\
\hline$\{3,4,5,6\}$ & $\left(\frac{16}{5}, 4\right]$ & $\left(\frac{16}{5}, \frac{14}{3}\right)$ \\
\hline
\end{tabular}

The lengthy proof of Theorem 6.7 can be found in the arXiv version:1803.10509.

\section{$7 \quad$ Final Remarks}

We conclude with some challenges for further possible research. The statement of Theorem 4.3 always requires $4 \in D$, but from Theorem 6.3 we know that there exist $D$-maxuniversal families of simple, 3-connected, 2-crossing-critical graphs for $D=\{3,5\}$ and $D=\{3,6\}$ (Figure $16 \mathrm{~b}$ ) and c)) e.g., when $4 \notin D$, and these can be generalized to any $k>2$ by a zip product with copies of $K_{3,3}$.

Hence it is an interesting open question of whether there exists a $D$-max-universal $k$ crossing-critical family such that $D \cap\{3,4\}=\varnothing$. It is unlikely that the answer would be easy since the question is related to another long standing open problem-whether there exists a 5 -regular $k$-crossing-critical infinite family. Related to this is the same question of existence of a 4-regular $k$-crossing-critical family, which does exist for $k=3$ [17] and the construction can be generalized to any $k \geqslant 6$, but the cases $k=4,5$ remain open.

Many more questions can be asked in a direct relation to the statement of Theorem 5.1, but we only mention a few of the most interesting ones. E.g., if $6 \notin D$, can the average degree of such a family be from the interval $[5,6)$ ? Or, assuming $3 \in D$ but $4 \notin D$, 
for which sets $D$ one can achieve $D$-max-universality and what are the related average degrees?

Concerning specifically 2-crossing-critical graphs, there are no open questions or cases left by the results of Section 6 . Yet, there is a natural open question related to Theorem 6.7, namely; how would the sets of admissible average degrees $I_{D}$ and $I_{D}^{s}$ change if we require all the vertex degrees in the constructed $D$-max-universal family to belong to $D$ ? There is a two-fold effect of this restriction. First, we would not be allowed to resolve the parity problem by adding an arbitrary small tile, and second, we could get some undesired degrees when joining two different tiles.

This question of precise degree set is nontrivial since, for example, in the case of simple graphs and $D=\{3,4\}$, we have only two tiles and there exist values of $r$ which actually force the total number of these tiles to be even (so our construction is not realizable). We leave this question open for further investigation.

We finish with another interesting structural conjecture:

Conjecture 7.1. There is a function $g: \mathbb{N} \rightarrow \mathbb{R}^{+}$such that, any sufficiently large simple 3 -connected $k$-crossing-critical graph has average degree greater than $3+g(k)$.

Note that corresponding result "on the upper side", i.e., bounding average degrees away from 6, has been established in [8]. Furthermore, note that the staircase strip generalization of Kochol's original implies $g\left(\left(\begin{array}{l}n \\ 2\end{array}\right)-1\right)<\frac{1}{4 n-7}$, cf. Theorem 3.2. The following problem therefore poses itself naturally:

Problem 7.2. Do staircase strips yield the sparsest $k$-crossing-critical graphs, ie. does there exist a $k$-crossing-critical family of graphs with average degree less than

$$
3+\frac{1}{2 \sqrt{1+8(k+1)}-5} ?
$$

\section{References}

[1] M. Ajtai, V. Chvátal, M. Newborn, and E. Szemerédi. Crossing-free subgraphs. In Theory and Practice of Combinatorics, volume 60 of North-Holland Mathematics Studies, pages 9 - 12. North-Holland, 1982.

[2] D. Bokal. On the crossing numbers of cartesian products with paths. J. Combin. Theory Ser. B, 97(3):381-384, 2007.

[3] D. Bokal. Infinite families of crossing-critical graphs with prescribed average degree and crossing number. J. Graph Theory, 65(2):139-162, 2010.

[4] D. Bokal, M. Chimani, and J. Leaños. Crossing number additivity over edge cuts. European J. Combin., 34(6):1010-1018, 2013.

[5] D. Bokal, B. Oporowski, R. B. Richter, and G. Salazar. Characterizing 2-crossingcritical graphs. Adv. in Appl. Math., 74:23-208, 2016. 
[6] Z. Dvořák and B. Mohar. Crossing-critical graphs with large maximum degree. J. Combin. Theory Ser. B, 100(4):413-417, 2010.

[7] J. F. Geelen, R. B. Richter, and G. Salazar. Embedding grids in surfaces. European J. Combin., 25(6):785-792, 2004.

[8] C. Hernández-Vélez, G. Salazar, and R. Thomas. Nested cycles in large triangulations and crossing-critical graphs. J. Combin. Theory Ser. B, 102(1):86-92, 2012.

[9] P. Hliněný. Crossing-critical graphs and path-width. In P. Mutzel, M. Jünger, and S. Leipert, editors, Graph Drawing, 9th International Symposium, GD 2001, Revised Papers, volume LNCS 2265 of Lecture Notes in Comput. Sci., pages 102-114. Springer, 2002.

[10] P. Hliněný. Crossing-number critical graphs have bounded path-width. J. Combin. Theory Ser. B, 88(2):347-367, 2003.

[11] P. Hliněný. New infinite families of almost-planar crossing-critical graphs. Electron. J. Combin., 15(1), \#R1022008.

[12] P. Hliněný and G. Salazar. Stars and bonds in crossing-critical graphs. J. Graph Theory, 65(3):198-215, 2010.

[13] M. Kochol. Construction of crossing-critical graphs. Discrete Math., 66(3):311-313, 1987.

[14] T. Leighton. Complexity Issues in VLSI. Foundations of Computing Series. MIT Press, Cambridge, MA, 1983.

[15] B. Pinontoan and R. B. Richter. Crossing numbers of sequences of graphs II: Planar tiles. J. Graph Theory, 42(4):332-341, 2003.

[16] B. Pinontoan and R. B. Richter. Crossing numbers of sequences of graphs I: General tiles. Australas. J. Combin., 30:197-206, 2004.

[17] R. B. Richter and C. Thomassen. Minimal graphs with crossing number at least $k$. J. Combin. Theory Ser. B, 58(2):217-224, 1993.

[18] G. Salazar. Infinite families of crossing-critical graphs with given average degree. Discrete Math., 271(1-3):343-350, 2003.

[19] J. Šrán̆. Infinite families of crossing-critical graphs with a given crossing number. Discrete Math., 48(1):129-132, 1984. 